\title{
Lepton mixing in gauge models
}

\author{
D. Falcone and L. Oliver \\ Laboratoire de Physique Théorique1 \\ Université de Paris XI, Bâtiment 210, 91405 Orsay Cedex, France
}

\begin{abstract}
We reexamine lepton mixing in gauge models by considering two theories within the type I seesaw mechanism, the Extended Standard Model, i.e. $S U(2)_{L} \times U(1)_{Y}$ with singlet right-handed heavy neutrinos, and the LeftRight Model $S U(2)_{L} \times S U(2)_{R} \times U(1)_{B-L}$. The former is often used as a simple heuristic approach to masses and mixing of light neutrinos and to leptogenesis, while we consider the latter as an introduction to other left-right symmetric gauge theories like $S O(10)$. We compare lepton mixing in both theories for general parameter space and discuss also some particular cases. In the electroweak broken phase, we study in parallel both models in the "current basis" (diagonal gauge interactions), and in the "mass basis" (diagonal mass matrices and mixing in the interaction), and perform the counting of $C P$ conserving and $C P$ violating parameters in both bases. We extend the analysis to the Pati-Salam model $S U(4)_{C} \times S U(2)_{L} \times S U(2)_{R}$ and to $S O(10)$. Although specifying the Higgs sector increases the predictive power, in the most general case one has the same parameter structure in the lepton sector for all the left-right symmetric gauge models. We make explicit the differences between the Extended Standard Model and the left-right models, in particular $C P$ violating and lepton-number violating new terms involving the $W_{R}$ gauge bosons. As expected, at low energy, the differences in the light neutrino spectrum and mixing appear only beyond leading order in the ratio of Dirac mass to right-handed Majorana mass.
\end{abstract}

\section{LPT-Orsay-14-41}

e-mails : domenicofalcone3@virgilio.it, Luis.Oliver@th.u-psud.fr

\footnotetext{
${ }^{1}$ Unité Mixte de Recherche UMR 8627 - CNRS
} 


\section{Introduction}

In the last years, an impressive experimental progress has been achieved on the neutrino spectrum and mixing. Using this information on the light neutrinos mass matrix $m_{L}$, one is tempted to use the inverse of the seesaw formula $M_{R}=-m_{D}^{t} m_{L}^{-1} m_{D}$, where $m_{D}$ is the Dirac neutrino mass matrix, as a window on high energy neutrino physics, i.e. on the heavy right-handed neutrino mass matrix $M_{R}$ [1, 2, 3, 4, 5].

To use the inverse seesaw formula one needs information on the crucial Dirac mass matrix $m_{D}$. It has been often suggested that theoretical information on this matrix can be guessed within the $S O(10)$ Grand Unification gauge theory [6]. In order to study the whole structure of $S O(10)$ as far as lepton mixing is concerned, we have realized that it is convenient to begin by considering simpler theories that also exhibit left-right (LR) symmetry (for a review, see ref. [7]).

The simplest gauge theory that has been builded to study lepton mixing is the one that we call Extended Standard Model (ESM), i.e. the Standard Model (SM) $S U(3) \times S U(2)_{L} \times U(1)_{Y}$ plus right-handed neutrinos $N_{R}$, one per generation, singlet under the SM gauge group. Although this scheme allows to introduce heavy righthanded neutrinos, it does not exhibit LR symmetry like $S O(10)$.

One main aim of the present paper is to compare lepton mixing in the ESM, on the one hand, with lepton mixing in left-right models like $S O(10)$. Lepton mixing in the ESM has been thoroughly studied in the literature [8, 9, 10, 11], specially in ref. [10] on which the present paper heavily relies, together with the comprehensive review paper [12].

To compare the ESM with left-right gauge theories we have found convenient to consider next the Left-Right Model $(\mathrm{LRM}) S U(2)_{L} \times S U(2)_{R} \times U_{B-L}(1)$ [13, 14], that exhibits a number of interesting new features concerning lepton mixing [15, 16]. This gauge group has already an appreciable complexity that will be useful as an introduction for the study of larger LR gauge groups, like the Pati-Salam model $S U(4)_{C} \times S U(2)_{L} \times S U(2)_{R}$ [17], and the grand unified $S O(10)$ gauge group [6].

We will first consider completely general Dirac or Majorana mass matrices consistent with Lorentz invariance, that coincide with mass matrices arising from the most general Higgs structure. We then look for the parameters that can be rotated away, although in a different way in the ESM and the LRM. We will consider the 
current basis, in which the interaction Lagrangian $\mathcal{L}_{w}$ is diagonal, and the mass basis, in which the mass Lagrangian $\mathcal{L}_{m}$ is diagonal, and we check that, for a given model, the final number of independent parameters, angles and phases, is the same in both bases.

Some main results exposed below are already known. The purpose of this paper is in part didactic, and in part the understanding a number of particular points. We think it is worth to explain in detail the differences between the Extended Standard model and the Left-Right gauge models as far as lepton mixing is concerned, specially the comparison of the interaction Lagrangians of both schemes in the mass basis.

Here below we expose briefly the fermion and gauge boson content of the ESM and LRM. In Sections 2 and 3 we perform the counting of the lepton sector parameters of the ESM and LRM in the current and in the mass bases. For the mass basis, special care is given to the approximation $m_{D}<<M_{R}$, as compared with exact results, and in Section 4 we recall two different representations proposed in the literature for the Dirac mass matrix $m_{D}$. In Section 5 we briefly examine leptogenesis in the ESM and in the LRM. In Section 6 we summarize the differences between both models for lepton mixing. Section 7 is devoted to the extension of our results to other left-right theories, Pati-Salam and $S O(10)$, and in Section 8 we conclude. In the Appendix we present some details of the calculations.

\subsection{Gauge boson and fermion content of the gauge models}

We now expose the fermion and gauge boson content of the two gauge theories that we consider in detail, the Extended Standard model and the Left-Right model $S U(2)_{L} \times S U(2)_{R} \times U(1)_{B-L}$.

\subsubsection{Extended Standard Model}

The Extended Standard Model (ESM) is just the Standard Model (SM) $S U(3) \times$ $S U(2)_{L} \times U(1)_{Y}$ with the addition of one Majorana fermion $N_{R}$ per generation, singlet under the gauge group. 
The fermion content of the model is for quarks

$$
\left(\begin{array}{c}
u_{L} \\
d_{L}
\end{array}\right) \sim\left(3,2, \frac{1}{3}\right), \quad u_{R} \sim\left(3,1, \frac{4}{3}\right), \quad d_{R} \sim\left(3,1,-\frac{2}{3}\right)
$$

and for leptons

$$
\left(\begin{array}{c}
\nu_{L} \\
e_{L}
\end{array}\right) \sim(\mathbf{1}, \mathbf{2},-1), \quad e_{R} \sim(\mathbf{1}, \mathbf{1},-2), \quad N_{R} \sim(\mathbf{1}, \mathbf{1}, 0)
$$

with

$$
Q=T_{3 L}+\frac{Y}{2}
$$

The gauge bosons are the gluons $(\mathbf{8}, \mathbf{1}, 0)$, the $W_{L}$ bosons $(\mathbf{1}, \mathbf{3}, 0)$ and the $B$ boson $(\mathbf{1}, \mathbf{1}, 0)$.

The Higgs sector needed to achieve the Spontaneous Symmetry Breaking (SSB) and give masses to the fermions is the usual doublet $\phi \sim(\mathbf{1}, \mathbf{2},-1)$. The novelty in the ESM with respect to the SM is just the presence of the Majorana $N_{R}$ singlet. The right-handed fermion $N_{R}$ can have a large mass, of a different scale than the SM, that can be originated from a Higgs boson, singlet relatively to the Standard Model $\Phi \sim(\mathbf{1}, \mathbf{1}, 0)$, or simply be a bare mass term

$$
(\mathbf{1}, \mathbf{1}, 0)_{f} \times(\mathbf{1}, \mathbf{1}, 0)_{f}=(\mathbf{1}, \mathbf{1}, 0)
$$

that, together with the Dirac mass terms

$$
(\mathbf{1}, \mathbf{2},-1)_{f} \times(\mathbf{1}, \mathbf{2}, 1)_{\bar{f}} \times(\mathbf{1}, \mathbf{2},-1)_{H}=(\mathbf{1}, \mathbf{1}, 0)+\ldots
$$

gives the general neutrino mass matrix

$$
\mathcal{M}=\left(\begin{array}{cc}
0 & m_{D} \\
m_{D}^{t} & M_{R}
\end{array}\right)
$$

where $m_{D}$ and $M_{R}$ are respectively general complex and complex symmetric matrices. 


\subsubsection{Left-Right Model}

In the LRM model $S U(3) \times S U(2)_{L} \times S U(2)_{R} \times U(1)_{B-L}$, the classification of L and $\mathrm{R}$ fermions is for quarks

$$
\left(\begin{array}{c}
u_{L} \\
d_{L}
\end{array}\right) \sim\left(3,2,1, \frac{1}{3}\right), \quad\left(\begin{array}{c}
u_{R} \\
d_{R}
\end{array}\right) \sim\left(3,1,2, \frac{1}{3}\right)
$$

and for leptons

$$
\left(\begin{array}{c}
\nu_{L} \\
e_{L}
\end{array}\right) \sim(\mathbf{1}, \mathbf{2}, \mathbf{1},-1), \quad\left(\begin{array}{c}
N_{R} \\
e_{R}
\end{array}\right) \sim(\mathbf{1}, \mathbf{1}, \mathbf{2},-1)
$$

with

$$
Q=T_{3 L}+T_{3 R}+\frac{B-L}{2}
$$

The gauge bosons are the gluons $(\mathbf{8}, \mathbf{1}, \mathbf{1}, 0)$, the $W_{L}$ bosons $(\mathbf{1}, \mathbf{3}, \mathbf{1}, 0)$, the $W_{R}$ bosons $(\mathbf{1}, \mathbf{1}, \mathbf{3}, 0)$ and the $B-L$ singlet $(\mathbf{1}, \mathbf{1}, \mathbf{1}, 0)$.

The Higgs fields needed to achieve SSB and the seesaw mechanism are the bidoublet $\phi \sim(\mathbf{1}, \mathbf{2}, \mathbf{2}, 0)$ and the triplet $\Delta_{R} \sim(\mathbf{1}, \mathbf{1}, \mathbf{3}, 2)$.

The bidoublet, written as

$$
\phi=\left(\begin{array}{ll}
\phi_{1}^{0} & \phi_{1}^{+} \\
\phi_{2}^{-} & \phi_{2}^{0}
\end{array}\right)
$$

breaks the SM group and gives masses to quarks and leptons through the Yukawa terms

$$
\begin{gathered}
\left(3,2,1, \frac{1}{3}\right)_{f} \times\left(\overline{3}, \mathbf{1}, 2,-\frac{1}{3}\right)_{\bar{f}} \times(\mathbf{1}, \mathbf{2}, \mathbf{2}, 0)_{H, \bar{H}}=(\mathbf{1}, \mathbf{1}, \mathbf{1}, 0)+\ldots \\
(\mathbf{1}, \mathbf{2}, \mathbf{1},-1)_{f} \times(\mathbf{1}, \mathbf{1}, \mathbf{2}, 1)_{\bar{f}} \times(\mathbf{1}, \mathbf{2}, \mathbf{2}, 0)_{H, \bar{H}}=(\mathbf{1}, \mathbf{1}, \mathbf{1}, 0)+\ldots
\end{gathered}
$$

with $H=\phi$ and $\bar{H}=\sigma_{2} H^{*} \sigma_{2}$.

From the vacuum expectation values

$$
<\phi_{1}^{0}>=k_{1}, \quad<\phi_{2}^{0}>=k_{2}
$$

that can be complex, the Yukawa couplings give the Dirac masses, as in the SM, but with a different pattern. Quark mass matrices $m_{u}, m_{d}$ and the Dirac neutrino mass matrix $m_{D}$ read

$$
m_{u}=p k_{1}+q k_{2}^{*}, \quad m_{d}=p k_{2}+q k_{1}^{*}
$$




$$
m_{D}=r k_{1}+s k_{2}^{*}, \quad m_{e}=r k_{2}+s k_{1}^{*}
$$

where $p, q, r$ and $s$ are complex Yukawa coupling matrices.

The triplet $H=\Delta_{R}$ breaks the LR model to the SM and, at the same time, gives a Majorana mass to the right-handed neutrino $N_{R}$ through the Yukawa term

$$
\begin{array}{cc}
(\mathbf{1}, \mathbf{1}, \mathbf{2},-1)_{f} \times(\mathbf{1}, \mathbf{1}, \mathbf{2},-1)_{f} \times(\mathbf{1}, \mathbf{1}, \mathbf{3}, 2)_{H}=(\mathbf{1}, \mathbf{1}, \mathbf{1}, 0)+\ldots \\
<\Delta_{R}^{0}>=v_{R}, & M_{R}=t v_{R},
\end{array}
$$

where $t$ is a complex symmetric Yukawa coupling matrix.

The full neutrino mass matrix has the form

$$
\mathcal{M}=\left(\begin{array}{cc}
0 & r k_{1}+s k_{2}^{*} \\
r^{t} k_{1}+s^{t} k_{2}^{*} & t v_{R}
\end{array}\right)
$$

i.e. it has the general form (마).

We consider this minimal Higgs content that is necessary in the LRM, and we do not introduce a possible left-handed triplet $\Delta_{L}=(\mathbf{1}, \mathbf{3}, \mathbf{1}, 2)_{H}$ that could in principle contribute to the light neutrino masses.

\section{Current basis}

In what follows, we consider the gauge models in the electroweak broken phase. We only make explicit the charged current terms in the interaction Lagragians of both gauge models.

\subsection{Extended Standard Model}

The mass and interaction Lagrangians write, in an obvious compact notation

$$
\begin{aligned}
& \mathcal{L}_{m}=\bar{\nu}_{L} m_{D} N_{R}+\frac{1}{2} \overline{\left(N_{R}\right)^{c}} M_{R} N_{R}+\bar{e}_{L} m_{e} e_{R}+\text { h.c. } \\
& \mathcal{L}_{w}=\bar{\nu}_{L} \gamma_{\mu} e_{L} W_{L}^{\mu}+\text { h.c. }
\end{aligned}
$$

The matrices $m_{D}$ and $m_{e}$ are general complex, each has 9 complex parameters, while $M_{R}$ is general complex symmetric with 6 complex parameters. 
The lepton number assignment $L\left(N_{R}\right)=-L\left(\left(N_{R}\right)^{c}\right)=1$ implies that the Majorana mass term is $|\Delta L|=2$ while, like for the other fermions, while the Dirac mass term is $|\Delta L|=0$.

From now on we adopt the following simplifying notation for the real parameters of an arbitrary square complex matrix $M$, that has $n(m)$ parameters, where $n$ is the total number of real parameters, among which there are $m(m \leq n)$ are phases :

$$
M \text { has } \mathrm{n}(\mathrm{m}) \text { real parameters } \leftrightarrow \mathrm{n} \text { real parameters, } \mathrm{m} \leq \mathrm{n} \text { phases }
$$

In this example, $m_{D}$ and $m_{e}$ have $18(9)$ real parameters and $M_{R}$ has $12(6)$ real parameters. Therefore, a priori one has in this model 30(15) real parameters.

Let us see now that we can reduce the number of independent parameters without modifying the interaction Lagrangian $\mathcal{L}_{w}$. Diagonalizing $m_{e}$ and $M_{R}$ by

$$
m_{e}=V_{e L}^{\dagger} m_{e}^{\text {diag }} V_{e R}, \quad M_{R}=U_{R}^{t} M_{R}^{\text {diag }} U_{R}
$$

and redefining the fields

$$
U_{R} N_{R} \rightarrow N_{R}, \quad \quad V_{e R} e_{R} \rightarrow e_{R}, \quad\left(\begin{array}{c}
V_{e L} \nu_{L} \\
V_{e L} e_{L}
\end{array}\right) \rightarrow\left(\begin{array}{c}
\nu_{L} \\
e_{L}
\end{array}\right)
$$

one gets

$$
\begin{aligned}
& \mathcal{L}_{m}=\bar{\nu}_{L} V_{e L} m_{D} U_{R}^{\dagger} N_{R}+\frac{1}{2} \overline{\left(N_{R}\right)^{c}} M_{R}^{\text {diag }} N_{R}+\bar{e}_{L} m_{e}^{\text {diag }} e_{R}+\text { h.c. } \\
& \mathcal{L}_{w}=\bar{\nu}_{L} \gamma_{\mu} e_{L} W_{L}^{\mu}+\text { h.c. }
\end{aligned}
$$

The simultaneous transformation of $\nu_{L}$ and $e_{L}$ in (20121) ensures the invariance of $\mathcal{L}_{w}$, but then $V_{e L}$ appears in the Dirac mass term. Since $m_{D}$ is a general complex symmetric matrix, so is $V_{e L} m_{D} U_{R}^{\dagger}$. Changing the notation

$$
V_{e L} m_{D} U_{R}^{\dagger} \rightarrow m_{D}
$$

one obtains

$$
\begin{aligned}
& \mathcal{L}_{m}=\bar{\nu}_{L} m_{D} N_{R}+\frac{1}{2} \overline{\left(N_{R}\right)^{c}} M_{R}^{\text {diag }} N_{R}+\bar{e}_{L} m_{e}^{\text {diag }} e_{R}+\text { h.c. } \\
& \mathcal{L}_{w}=\bar{\nu}_{L} \gamma_{\mu} e_{L} W_{L}^{\mu}+\text { h.c. }
\end{aligned}
$$


We can redefine the doublet $\left(\begin{array}{c}\nu_{L} \\ e_{L}\end{array}\right)$ and the singlet $e_{R}$ by the same diagonal phase matrix $P_{e}$ :

$$
\left(\begin{array}{c}
\nu_{L} \\
e_{L}
\end{array}\right) \rightarrow\left(\begin{array}{c}
P_{e} \nu_{L} \\
P_{e} e_{L}
\end{array}\right), \quad \quad e_{R} \rightarrow P_{e} e_{R}
$$

and one gets

$$
\begin{aligned}
& \mathcal{L}_{m}=\bar{\nu}_{L} P_{e}^{*} m_{D} N_{R}+\frac{1}{2} \overline{\left(N_{R}\right)^{c}} M_{R}^{\text {diag }} N_{R}+\bar{e}_{L} m_{e}^{\text {diag }} e_{R}+\text { h.c. } \\
& \mathcal{L}_{w}=\bar{\nu}_{L} \gamma_{\mu} e_{L} W_{L}^{\mu}+\text { h.c. }
\end{aligned}
$$

Finally we can choose the phase matrix $P_{e}$ to cancel three phases of $m_{D}$ in $P_{e}^{*} m_{D}$ :

$$
\begin{aligned}
& \mathcal{L}_{m}=\bar{\nu}_{L} m_{D} N_{R}+\frac{1}{2} \overline{\left(N_{R}\right)^{c}} M_{R}^{\text {diag }} N_{R}+\bar{e}_{L} m_{e}^{\text {diag }} e_{R}+\text { h.c. } \\
& \mathcal{L}_{w}=\bar{\nu}_{L} \gamma_{\mu} e_{L} W_{L}^{\mu}+\text { h.c. }
\end{aligned}
$$

where now the Dirac mass matrix $m_{D}$ is not a general complex matrix, but has 9 real parameters +6 phases, i.e. 15(6) real parameters.

To summarize parameter counting, one is left in the current basis with 15(6) $\left(\right.$ from $\left.m_{D}\right)+3(0)\left(\right.$ from $\left.m_{e}^{\text {diag }}\right)+3(0)\left(\right.$ from $\left.M_{R}^{\text {diag }}\right)=21(6)$ real parameters, i.e. among them 6 phases. This counting agrees with the one performed in ref. [18].

\subsection{Left-Right Model}

In the LRM, the Lagrangian in the lepton sector writes

$$
\begin{aligned}
& \mathcal{L}_{m}=\bar{\nu}_{L} m_{D} N_{R}+\frac{1}{2} \overline{\left(N_{R}\right)^{c}} M_{R} N_{R}+\bar{e}_{L} m_{e} e_{R}+\text { h.c. } \\
& \mathcal{L}_{w}=\bar{\nu}_{L} \gamma_{\mu} e_{L} W_{L}^{\mu}+\bar{N}_{R} \gamma_{\mu} e_{R} W_{R}^{\mu}+\text { h.c. }
\end{aligned}
$$

Notice that, to simplify the notation, possible $W_{L}-W_{R}$ mixing is for the moment neglected in the interaction term, that will be considered later. The matrices $m_{D}$ and $m_{e}$ are a priori general complex with 18(9) parameters each, and $M_{R}$ is a general complex symmetric matrix with 12(6) parameters.

An important remark is in order here. Parameter counting of the Left-Right Model in the "Current basis" means that we are assuming the whole interaction 
Lagrangian $\mathcal{L}_{w}$ in (27) to be diagonal, both in the left and the right sectors. For low energy neutrino physics, it can seem academic to assume that the right-handed piece $\bar{N}_{R} \gamma_{\mu} e_{R} W_{R}^{\mu}+$ h.c. is kept diagonal, because it is an interaction term involving high scale degrees of freedom. However, this natural assumption in any LR gauge theory is not only a formal point since, to keep this piece diagonal amounts to assume that one assigns a lepton number to the $N_{R}$ neutrinos, just in the same way as it is done for the $\nu_{L}$ neutrinos in (27), and in consistency with the assignment $L\left(N_{R}\right)=-L\left(\left(N_{R}\right)^{c}\right)=1$ in the ESM. As we will see below, the diagonalization of the light neutrino mass matrix and of the right neutrino mass matrix will result in mixing matrices of the PMNS type for both the light and the heavy neutrinos.

Diagonalizing $m_{e}$ by (19) and redefining the fields

$$
\left(\begin{array}{c}
V_{e L} \nu_{L} \\
V_{e L} e_{L}
\end{array}\right) \rightarrow\left(\begin{array}{c}
\nu_{L} \\
e_{L}
\end{array}\right), \quad\left(\begin{array}{c}
V_{e R} N_{R} \\
V_{e R} e_{R}
\end{array}\right) \rightarrow\left(\begin{array}{c}
N_{R} \\
e_{R}
\end{array}\right)
$$

one gets

$$
\begin{aligned}
\mathcal{L}_{m} & =\bar{\nu}_{L} V_{e L} m_{D} V_{e R}^{\dagger} N_{R}+\frac{1}{2} \overline{\left(N_{R}\right)^{c}} V_{e R}^{*} M_{R} V_{e R}^{\dagger} N_{R}+\bar{e}_{L} m_{e}^{\text {diag }} e_{R}+\text { h.c. } \\
\mathcal{L}_{w} & =\bar{\nu}_{L} \gamma_{\mu} e_{L} W_{L}^{\mu}+\bar{N}_{R} \gamma_{\mu} e_{R} W_{R}^{\mu}+\text { h.c. }
\end{aligned}
$$

Since $m_{D}$ is general complex, so is $V_{e L} m_{D} V_{e R}^{\dagger}$, and $M_{R}$ being complex symmetric, so is $V_{e R}^{*} M_{R} V_{e R}^{\dagger}$.

Changing the notation

$$
V_{e L} m_{D} V_{e R}^{\dagger} \rightarrow m_{D}, \quad V_{e R}^{*} M_{R} V_{e R}^{\dagger} \rightarrow M_{R}
$$

one obtains

$$
\begin{aligned}
\mathcal{L}_{m} & =\bar{\nu}_{L} m_{D} N_{R}+\frac{1}{2} \overline{\left(N_{R}\right)^{c}} M_{R} N_{R}+\bar{e}_{L} m_{e}^{\text {diag }} e_{R}+\text { h.c. } \\
\mathcal{L}_{w} & =\bar{\nu}_{L} \gamma_{\mu} e_{L} W_{L}^{\mu}+\bar{N}_{R} \gamma_{\mu} e_{R} W_{R}^{\mu}+\text { h.c. }
\end{aligned}
$$

We can redefine the doublets by the same diagonal phase matrix $P_{e}$ :

$$
\left(\begin{array}{c}
\nu_{L} \\
e_{L}
\end{array}\right) \rightarrow\left(\begin{array}{c}
P_{e} \nu_{L} \\
P_{e} e_{L}
\end{array}\right), \quad\left(\begin{array}{c}
N_{R} \\
e_{R}
\end{array}\right) \rightarrow\left(\begin{array}{c}
P_{e} N_{R} \\
P_{e} e_{R}
\end{array}\right)
$$


and one gets

$$
\begin{aligned}
\mathcal{L}_{m} & =\bar{\nu}_{L} P_{e}^{*} m_{D} P_{e} N_{R}+\frac{1}{2} \overline{\left(N_{R}\right)^{c}} P_{e} M_{R} P_{e} N_{R}+\bar{e}_{L} m_{e}^{\text {diag }} e_{R}+\text { h.c. } \\
\mathcal{L}_{w} & =\bar{\nu}_{L} \gamma_{\mu} e_{L} W_{L}^{\mu}+\bar{N}_{R} \gamma_{\mu} e_{R} W_{R}^{\mu}+h . c .
\end{aligned}
$$

We can chose the phase matrix $P_{e}$ to cancel three phases of $m_{D}$ or three phases of $M_{R}$, but not both at the same time. We choose to absorb 3 phases in $M_{R}$. Changing the notation $P_{e}^{*} m_{D} P_{e} \rightarrow m_{D}$, one gets finally

$$
\begin{aligned}
& \mathcal{L}_{m}=\bar{\nu}_{L} m_{D} N_{R}+\frac{1}{2} \overline{\left(N_{R}\right)^{c}} M_{R} N_{R}+\bar{e}_{L} m_{e}^{\text {diag }} e_{R}+\text { h.c. } \\
& \mathcal{L}_{w}=\bar{\nu}_{L} \gamma_{\mu} e_{L} W_{L}^{\mu}+\bar{N}_{R} \gamma_{\mu} e_{R} W_{R}^{\mu}+\text { h.c. }
\end{aligned}
$$

where $m_{D}$ is an arbitrary complex matrix with 18(9) parameters and $M_{R}$ is complex symmetric with $9(3)$ parameters.

To summarize, one gets finally in the LRM : 18(9) parameters from $m_{D}+9(3)$ parameters from $M_{R}+3$ eigenvalues in $m_{e}^{\text {diag }}=30(12)$ parameters.

Much more constrained models have been considered in the literature. For example, the Minimal LRM within supersymmetry with a Higgs content that implies $m_{e}=m_{D}, m_{u}=m_{d}$ (up-down unification) [19], that has a reduced number of parameters.

\section{Mass basis}

\subsection{Extended Standard Model}

For the diagonalization of the whole $6 \times 6$ neutrino mass matrix, we proceed step by step, and we begin with (26), where $m_{e}^{\text {diag }}$ and $M_{R}^{\text {diag }}$ are diagonal and the Dirac mass matrix $m_{D}$ has $15(6)$ parameters. So we can rewrite

$$
\begin{aligned}
\mathcal{L}_{m} & =\frac{1}{2}\left(\overline{\nu_{L}}, \overline{\left(N_{R}\right)^{c}}\right) \mathcal{M}\left(\begin{array}{c}
\nu_{L}^{c} \\
N_{R}
\end{array}\right)+\bar{e}_{L} m_{e}^{\text {diag }} e_{R}+\text { h.c. } \\
\mathcal{L}_{w} & =\bar{\nu}_{L} \gamma_{\mu} e_{L} W_{L}^{\mu}+h . c .
\end{aligned}
$$

where $\mathcal{M}$ has the form

$$
\mathcal{M}=\left(\begin{array}{cc}
0 & m_{D} \\
m_{D}^{t} & M_{R}^{\text {diag }}
\end{array}\right)
$$


This matrix has $18(6)$ parameters : $15(6)$ from $m_{D}$ and $3(0)$ from $M_{R}^{\text {diag }}$.

Let us now diagonalize $\mathcal{M}$ with the unitary matrix $V$ [9, 10, 11]

$$
\mathcal{M}=V \mathcal{M}^{\text {diag }} V^{t}
$$

where

$$
\begin{gathered}
\mathcal{M}^{\text {diag }}=\left(\begin{array}{cc}
m_{L}^{\text {diag }} & 0 \\
0 & M_{R}^{\text {diag }}
\end{array}\right) \\
V=\left(\begin{array}{cc}
K & R \\
S & T
\end{array}\right)
\end{gathered}
$$

Notice that since $\mathcal{M}^{\text {diag }}$ has 6 eigenvalues, and $\mathcal{M}$ has $18(6)$ parameters, the $6 \times 6$ unitary matrix $V$ will have $18(6)$ - $6(0=12(6)$ parameters. Rewriting (35) under the form

$$
\begin{aligned}
& \mathcal{L}_{m}=\frac{1}{2}\left(\overline{\nu_{L}}, \overline{\left(N_{R}\right)^{c}}\right) V \mathcal{M}^{\text {diag }} V^{t}\left(\begin{array}{c}
\nu_{L}^{c} \\
N_{R}
\end{array}\right)+\bar{e}_{L} m_{e}^{\text {diag }} e_{R}+\text { h.c. } \\
& \mathcal{L}_{w}=\left(\overline{\nu_{L}}, \overline{\left(N_{R}\right)^{c}}\right) \gamma_{\mu}\left(\begin{array}{cc}
1 & 0 \\
0 & 0
\end{array}\right)\left(\begin{array}{c}
e_{L} \\
e_{L}
\end{array}\right) W_{L}^{\mu}+\text { h.c. }
\end{aligned}
$$

and redefining

$$
V^{t}\left(\begin{array}{c}
\nu_{L}^{c} \\
N_{R}
\end{array}\right) \rightarrow\left(\begin{array}{c}
\nu_{L}^{c} \\
N_{R}
\end{array}\right), \quad\left(\bar{\nu}, \overline{\left(N_{R}\right)^{c}}\right) V \rightarrow\left(\bar{\nu}, \overline{\left(N_{R}\right)^{c}}\right)
$$

one gets

$$
\begin{aligned}
& \mathcal{L}_{m}=\frac{1}{2}\left(\overline{\nu_{L}}, \overline{\left(N_{R}\right)^{c}}\right) \mathcal{M}^{\text {diag }}\left(\begin{array}{c}
\nu_{L}^{c} \\
N_{R}
\end{array}\right)+\bar{e}_{L} m_{e}^{\text {diag }} e_{R}+\text { h.c. } \\
& \mathcal{L}_{w}=\left(\overline{\nu_{L}}, \overline{\left(N_{R}\right)^{c}}\right) \gamma_{\mu} V^{\dagger}\left(\begin{array}{cc}
1 & 0 \\
0 & 0
\end{array}\right)\left(\begin{array}{c}
e_{L} \\
e_{L}
\end{array}\right) W_{L}^{\mu}+\text { h.c. }
\end{aligned}
$$

or

$$
\begin{aligned}
\mathcal{L}_{m} & =\frac{1}{2} \overline{\nu_{L}} m_{L}^{\text {diag }}\left(\nu_{L}\right)^{c}+\frac{1}{2} \overline{\left(N_{R}\right)^{c}} M_{R}^{\text {diag }} N_{R}+\bar{e}_{L} m_{e}^{\text {diag }} e_{R}+\text { h.c. } \\
\mathcal{L}_{w} & =\left(\overline{\nu_{L}} K^{\dagger} e_{L}+\overline{\left(N_{R}\right)^{c}} R^{\dagger} e_{L}\right) \gamma_{\mu} W_{L}^{\mu}+h . c .
\end{aligned}
$$


The first term in $\mathcal{L}_{w}$ describes the Standard Model $\Delta L=0$ decay

$$
W_{L} \rightarrow e_{L} \bar{\nu}_{L}
$$

while the second term corresponds to the well-known $\Delta L=2$ process

$$
\left(N_{R}\right)^{c} \rightarrow e_{L} W_{L}
$$

$\left(L\left(N_{R}\right)=-L\left(\left(N_{R}\right)^{c}\right)=L\left(e_{L}\right)\right)$. The notation $\left(N_{R}\right)^{c}$ for the heavy neutrino makes explicit also the chirality conservation of the $V-A$ interaction.

Notice that only the $3 \times 3$ complex matrices $K$ and $R$ from the $6 \times 6$ unitary matrix (39) are involved in the formula (43). Let us now count the parameters of these matrices. From the zero in the matrix $\mathcal{M}(36)$ and the definitions (37, 39) one finds (see eqn. (144) of the Appendix for $m_{L}=0$ )

$$
K m_{L}^{\text {diag }} K^{t}+R M_{R}^{\text {diag }} R^{t}=0
$$

Using the unitarity of the matrix $V$ (39) one has

$$
K K^{\dagger}+R R^{\dagger}=1
$$

Eqns. (46) and (47) are identities between $3 \times 3$ matrices involving only the mixing matrices $K$ and $R$ and not the whole matrix (39). Due to these relations, the matrices $K$ and $R$ are correlated.

The conditions (46) and (47) reduce the number of independent parameters. Equation (46) is self-transposed, and gives 12(6) constraints, while (47) is hermitian, giving $9(3)$ constraints. This reduces the number of parameters of the two complex matrices $K$ and $R$ from 36(18) down to $15(9)$.

Finally, redefining the charged lepton fields by a diagonal $3 \times 3$ phase matrix $Q_{e}$

$$
e_{L} \rightarrow Q_{e}^{\dagger} e_{L}, \quad e_{R} \rightarrow Q_{e}^{\dagger} e_{R}
$$

one gets, from (43),

$$
\begin{aligned}
& \mathcal{L}_{m}=\frac{1}{2} \overline{\nu_{L}} m_{L}^{\text {diag }}\left(\nu_{L}\right)^{c}+\frac{1}{2} \overline{\left(N_{R}\right)^{c}} M_{R}^{\text {diag }} N_{R}+\bar{e}_{L} m_{e}^{\text {diag }} e_{R}+\text { h.c. } \\
& \mathcal{L}_{w}=\left(\overline{\nu_{L}}\left(Q_{e} K\right)^{\dagger}+\overline{\left(N_{R}\right)^{c}}\left(Q_{e} R\right)^{\dagger}\right) \gamma_{\mu} e_{L} W_{L}^{\mu}+h . c
\end{aligned}
$$


On the other hand, multiplying (46) on the left by $Q_{e}$ and on the right by $Q_{e}^{t}$, and (477) on the left by $Q_{e}$ and on the right by $Q_{e}^{\dagger}$, these equations become

$$
\begin{gathered}
\left(Q_{e} K\right) m_{L}^{\text {diag }}\left(Q_{e} K\right)^{t}+\left(Q_{e} R\right) M_{R}^{\text {diag }}\left(Q_{e} R\right)^{t}=0 \\
\left(Q_{e} K\right)\left(Q_{e} K\right)^{\dagger}+\left(Q_{e} R\right)\left(Q_{e} R\right)^{\dagger}=1
\end{gathered}
$$

and we can absorb 3 phases of one of the matrices $K$ or $R$, but not of both matrices at the same time.

In summary, the matrices $K$ and $R$ have together 12(6) parameters, and adding the 9(0) parameters from $m_{e}^{\text {diag }}, m_{L}^{\text {diag }}$ and $M_{R}^{\text {diag }}$ one obtains a total of 21(6) parameters, the same number as in the current basis. In the ESM the matrices $K$ and $R$ are decoupled from $S$ and $T$ of (39), and obey relations (46,47).

We can now go somewhat further by considering first the whole matrix (39), and assuming $m_{D}<<M_{R}$.

\subsubsection{The matrices $K, R, S, T$ in the Extended Standard Model}

Starting from the Lagrangian in the current basis (26), $m_{D}$ has now 15(6) parameters. Particularizing formulas (144,146) of the Appendix to the present case, we have :

$$
\begin{aligned}
& K m_{L}^{\text {diag }} K^{t}+R M_{R}^{\text {diag }} R^{t}=0 \\
& S m_{L}^{\text {diag }} S^{t}+T M_{R}^{\text {diag }} T^{t}=M_{R}^{\text {diag }} \\
& K m_{L}^{\text {diag }} S^{t}+R M_{R}^{\text {diag }} T^{t}=m_{D}
\end{aligned}
$$

Considering for the moment the unitarity of the matrix (39), the number of independent parameters in the l.h.s. will be 36(21) from $(K, R, S, T)+3(0)$ from $m_{L}^{\text {diag }}+3(0)$ from $M_{R}^{\text {diag }}=42(21)$.

The complex symmetric matrix equation (52) gives 12(6) constraints. On the other hand, $M_{R}^{\text {diag }}$ appears already in the r.h.s. of (53) , and this equation implies $12(6)-3(0)=9(6)$ constraints. Since $m_{D}$ has now $15(6)$ free parameters, eq. (54) gives 3(3) constraints, giving a total of $12(6)+9(6)+3(3)=24(15)$ constraints. Therefore the number of independent parameters is $42(21)-24(15)=18(6)$ parameters. Adding the 3(0) eigenvalues of $m_{e}^{\text {diag }}$ one gets $18(6)+3(0)=21(6)$ parameters, the same result as in the current basis. 
Moreover, substracting from this total of 21(6) parameters the 9(0) mass eigenvalues $m_{e}^{\text {diag }}, m_{L}^{\text {diag }}$ and $M_{R}^{\text {diag }}$, the set of matrices $(K, R, S, T)$ has $12(6)$ parameters, the same number that we have found for $K$ and $R$, so that $S$ and $T$ are not independent.

Exact relations between the matrices $K, R, S, T$

On the other hand, from (36 39 ) one has

$$
\left(\begin{array}{cc}
0 & m_{D} \\
m_{D}^{t} & M_{R}^{\text {diag }}
\end{array}\right)\left(\begin{array}{cc}
K^{*} & R^{*} \\
S^{*} & T^{*}
\end{array}\right)=\left(\begin{array}{cc}
K & R \\
S & T
\end{array}\right)\left(\begin{array}{cc}
m_{L}^{\text {diag }} & 0 \\
0 & M_{R}^{\text {diag }}
\end{array}\right)
$$

hence

$$
\left(\begin{array}{cc}
m_{D} S^{*} & m_{D} T^{*} \\
m_{D}^{t} K^{*}+M_{R}^{\text {diag }} S^{*} & m_{D}^{t} R^{*}+M_{R}^{\text {diag }} T^{*}
\end{array}\right)=\left(\begin{array}{cc}
K m_{L}^{\text {diag }} & R M_{R}^{\text {diag }} \\
S m_{L}^{\text {diag }} & T M_{R}^{\text {diag }}
\end{array}\right)
$$

and therefore one obtains the following exact expressions of the matrices $R, S$ in terms of $K, T, m_{D}$ and the mass eigenvalues :

$$
\begin{aligned}
& R=m_{D} T^{*}\left(M_{R}^{\text {diag }}\right)^{-1} \\
& S=\left(m_{D}^{*}\right)^{-1} K^{*} m_{L}^{\text {diag }}
\end{aligned}
$$

From inspection of the precedent equations, one sees that (57,58) are relations between the mass basis quantities $\left(K, R, S, T, m_{L}^{\text {diag }}, M_{R}^{\text {diag }}\right)$ and the current basis matrices $m_{D}, M_{R}^{\text {diag }}$, since $M_{R}$ is diagonalized and appears in both bases. Eliminating $m_{D}$, one finds an exact relation between quantities in the mass basis :

$$
M_{R}^{\text {diag }} T^{-1} S=\left(R^{*}\right)^{-1} K^{*} m_{L}^{\operatorname{diag}}
$$

The matrices $(K, R, S, T)$ for $m_{D}<<M_{R}$

If $m_{D}<<M_{R}$, one has the order of magnitude

$$
R \sim S \sim O\left(\frac{m_{D}}{M_{R}}\right)
$$

Neglecting in equations $\left(138,[143)\right.$ of the Appendix the terms of $O\left(\frac{m_{D}^{2}}{M_{R}^{2}}\right)$ one gets the approximate unitarity conditions

$$
K K^{\dagger} \simeq K^{\dagger} K \simeq 1
$$




$$
T T^{\dagger} \simeq T^{\dagger} T \simeq 1
$$

Moreover, from (61,62), both equations (140) and (143) imply the same approximate relation between $R$ and $S$

$$
R \simeq-K S^{\dagger} T
$$

In conclusion, in the present approximation one gets two unitary matrices $K$ and $T$ (61,62) and the matrix $R$ given in terms of $(K, T, S)$ by (63).

On the other hand, neglecting terms of $O\left(\frac{m_{D}^{2}}{M_{R}^{2}}\right)$ in (52+54), one gets

$$
\begin{aligned}
& K m_{L}^{\text {diag }} K^{t}+R M_{R}^{\text {diag }} R^{t}=0 \\
& T M_{R}^{\text {diag }} T^{t} \simeq M_{R}^{\text {diag }} \\
& R M_{R}^{\text {diag }} T^{t} \simeq m_{D}
\end{aligned}
$$

Eqn. (65) implies

$$
T \simeq 1
$$

Notice that (66) is identical to the relation (57) obtained above. On the other hand, combining (63) with the exact relation (59) one consistently obtains obtains (64).

One can see that (63) gives just the seesaw formula. From (57, 58, 67), eqn. (63) implies, after some algebra

$$
K m_{L}^{\text {diag }} K^{t} \simeq-m_{D}\left(M_{R}^{\text {diag }}\right)^{-1} m_{D}^{t}
$$

and from the general complex symmetric matrix $m_{L}$,

$$
m_{L}=K m_{L}^{\text {diag }} K^{t}
$$

one gets the seesaw formula in the ESM :

$$
m_{L} \simeq-m_{D}\left(M_{R}^{\text {diag }}\right)^{-1} m_{D}^{t}
$$

We see that $K$ is the mixing matrix for light neutrinos, that appears in (43) in the basis in which $m_{e}$ is diagonal.

On the other hand, relation (57) or (66), together with (67), implies

$$
R \simeq m_{D}\left(M_{R}^{\text {diag }}\right)^{-1}
$$


and using the seesaw formula (70), relation (58) becomes

$$
S=-\left(M_{R}^{\text {diag }}\right)^{-1} m_{D}^{\dagger} K
$$

in consistency with (63).

The whole set $K, R, S, T$ has 12(6) parameters, implying from (67) that $K, R$ and $S$ have 12(6) independent parameters. Since according to (72) the matrix $S$ is not independent, the matrices $K, R$ that appear in the interaction Lagrangian (43), have together 12(6) parameters. From (71) and the 15(6) number of parameters of $m_{D}$, we see that $R$ will have $12(6)$ parameters. Since $K$ is unitary in the present approximation, we can choose 6(3) independent parameters within $R$ to provide the unitary matrix $K$ with $6(3)$ parameters, the physically relevant PMNS structure. Then $R$ will have other extra 6(3) parameters. However, other solutions are allowed, since $K$ is unitary, not necessarily of the PMNS type.

\subsubsection{Summary of the parameter counting in the mass basis}

In the mass basis, parameter counting in the physically relevant case is : $12(6)$ parameters from both the complex matrices $K, R$ (among these, 6(3) parameters from the PMNS-like matrix $\mathrm{K})+3(0)$ parameters from $M_{R}^{\text {diag }}+3(0)$ parameters

from $m_{L}^{\text {diag }}+3(0)$ parameters from $m_{e}^{\text {diag }}=21(6)$, the same counting as in the current basis.

The more constrained condition $m_{D}<<M_{R}$ provides a particular case : $R$ has 12(6) parameters, among which one has to choose the 6(3) parameters of the PMNS matrix $K$.

\subsection{Left-Right Model}

Let us start from the Lagrangian (34) of the LRM. At this stage $M_{R}$ is complex symmetric with $9(3)$ parameters. We rewrite (34) under the form

$$
\begin{gathered}
\mathcal{L}_{m}=\frac{1}{2}\left(\overline{\nu_{L}}, \overline{\left(N_{R}\right)^{c}}\right) \mathcal{M}\left(\begin{array}{c}
\left(\nu_{L}\right)^{c} \\
N_{R}
\end{array}\right)+\bar{e}_{L} m_{e}^{\text {diag }} e_{R}+\text { h.c. } \\
\mathcal{L}_{w}=\left(\overline{\nu_{L}}, \overline{\left(N_{R}\right)^{c}}\right) \gamma_{\mu}\left(\begin{array}{cc}
1 & 0 \\
0 & 0
\end{array}\right)\left(\begin{array}{c}
e_{L} \\
e_{L}
\end{array}\right) W_{L}^{\mu}
\end{gathered}
$$




$$
+\left(\overline{N_{R}}, \overline{\left(\nu_{L}\right)^{c}}\right) \gamma_{\mu}\left(\begin{array}{ll}
1 & 0 \\
0 & 0
\end{array}\right)\left(\begin{array}{c}
e_{R} \\
e_{R}
\end{array}\right) W_{R}^{\mu}+\text { h.c. }
$$

where

$$
\mathcal{M}=\left(\begin{array}{cc}
0 & m_{D} \\
m_{D}^{t} & M_{R}
\end{array}\right)
$$

Unlike the case of the ESM, the complex symmetric block $M_{R}$ is not diagonalized, it has $9(3)$ parameters since three phases have been rotated away.

Using the unitary matrix $V$ (36+39),

$$
\begin{aligned}
& \left(\overline{\nu_{L}}, \overline{\left(N_{R}\right)^{c}}\right) \rightarrow\left(\overline{\nu_{L}}, \overline{\left(N_{R}\right)^{c}}\right) V^{\dagger} \\
& \left(\overline{N_{R}}, \overline{\left(\nu_{L}\right)^{c}}\right) \rightarrow\left(\overline{N_{R}}, \overline{\left(\nu_{L}\right)^{c}}\right)\left(\begin{array}{ll}
0 & 1 \\
1 & 0
\end{array}\right) V^{t}\left(\begin{array}{ll}
0 & 1 \\
1 & 0
\end{array}\right)
\end{aligned}
$$

we obtain the following Lagrangian in the mass basis

$$
\begin{aligned}
& \mathcal{L}_{m}=\frac{1}{2} \overline{\nu_{L}} m_{L}^{\text {diag }}\left(\nu_{L}\right)^{c}+\frac{1}{2} \overline{\left(N_{R}\right)^{c}} M_{R}^{\text {diag }} N_{R}+\bar{e}_{L} m_{e}^{\text {diag }} e_{R}+\text { h.c. } \\
& \mathcal{L}_{w}=\left(\overline{\nu_{L}} K^{\dagger}+\overline{\left(N_{R}\right)^{c}} R^{\dagger}\right) \gamma_{\mu} e_{L} W_{L}^{\mu}+\left(\overline{N_{R}} T^{t}+\overline{\left(\nu_{L}\right)^{c}} S^{t}\right) \gamma_{\mu} e_{R} W_{R}^{\mu}+\text { h.c. }
\end{aligned}
$$

The $3 \times 3$ matrices $K$ and $R$ enter in the left sector, while $T$ and $S$ enter in the right sector, in a symmetric way. A formula of similar structure to eqn. (176) follow from the results of ref. [16], that uses however a quite different notation.

It is important to point out that the terms dependent on $K$ and $T$ are lepton number conserving $\Delta L=0$, while those that depend on $R$ and $S$ are lepton number violating $\Delta L=2$.

\subsubsection{The matrices $K, R, S, T$ in the Left-Right Model}

Particularizing (144,146) of the Appendix to the case (174), we have :

$$
\begin{aligned}
& K m_{L}^{\text {diag }} K^{t}+R M_{R}^{\text {diag }} R^{t}=0 \\
& S m_{L}^{\text {diag }} S^{t}+T M_{R}^{\text {diag }} T^{t}=M_{R} \\
& K m_{L}^{\text {diag }} S^{t}+R M_{R}^{\text {diag }} T^{t}=m_{D}
\end{aligned}
$$

Considering for the moment only the unitarity of the full matrix $V$ (39), that has 36(21) parameters, the number of independent parameters in the 1.h.s. of the 
precedent equations will be $36(21)$ from $(K, R, S, T)+3(0)+$ from $m_{L}^{\text {diag }}+3(0)$ from $M_{R}^{\text {diag }}=42(21)$ parameters.

The complex symmetric matrix equation (77) gives 12(6) constraints. On the other hand, $M_{R}$ in the r.h.s. of (78) has $9(3)$ free parameters, and this equation implies $12(6)-9(3)=3(3)$ constraints. Finally, since $m_{D}$ is a general complex matrix, with 18(9) free parameters, eq. (79) does not give any constraint. This gives a total of $12(6)+3(3)=15(9)$ constraints. Therefore one has $42(21)-15(9)=$ 27(12) independent parameters. Adding the 3(0) eigenvalues of $m_{e}^{\text {diag }}$, not counted up to now, one gets $27(12)+3(0)=30(12)$ parameters, the same result as in the current basis.

Moreover, substracting from this total number of 30(12) parameters the $9(0)$ mass eigenvalues $m_{e}^{\text {diag }}, m_{L}^{\text {diag }}$ and $M_{R}^{\text {diag }}$, we see that the set of matrices $(K, R, S, T)$, that appear in the interaction term (76) , have a total of 21(12) parameters.

In the $S U(2)_{L} \times S U(2)_{R} \times U(1)$ Model one obtains also the exact relations between the matrices $K, R, S, T$ given above by eqns. (55.59).

The matrices $(K, R, S, T)$ for $m_{D}<<M_{R}$

The relations given above within the approximation $m_{D}<<M_{R}$ (61-63) for the ESM also hold in the LR model.

Let us rewrite eqns. (777,79) neglecting terms of $O\left(\frac{m_{D}^{2}}{M_{R}^{2}}\right)$ :

$$
\begin{aligned}
& K m_{L}^{\text {diag }} K^{t}+R M_{R}^{\text {diag }} R^{t}=0 \\
& T M_{R}^{\text {diag }} T^{t} \simeq M_{R} \\
& R M_{R}^{\text {diag }} T^{t} \simeq m_{D}
\end{aligned}
$$

Eqn. (82) is the above obtained exact relation (57) if one neglects in the latter higher order terms. This means that in (79), the first term of the l.h.s., that is of $O\left(m_{D}^{3} / M_{R}^{2}\right)$, is compensated by higher order terms in the second term $R M_{R}^{d i a g} T^{t}$. On the other hand, combining (63) with the exact relation (59), one consistently obtains the exact relation (80).

According to (62) and (81), the matrix $T$ is the unitary mixing matrix of righthanded neutrinos, for which we can take 6(3) parameters, i.e. a matrix of the PMNS 
type. Eqn. (61) holds also in the LRM, and $K$ is the unitary mixing matrix of light left-handed neutrinos.

Since the whole set $K, R, S$ and $T$ has 21(12) parameters and the matrices $K, T$ have 6(3) parameters each, this implies that $R$ and $S$ can have together $9(6)$ extra independent parameters.

In the LR model, from relations (63) and (81) one obtains

$$
K m_{L}^{\text {diag }} K^{t} \simeq-m_{D} T^{*}\left(M_{R}^{\text {diag }}\right)^{-1} T^{\dagger} m_{D}^{t}
$$

i.e. the seesaw formula

$$
m_{L} \simeq-m_{D} M_{R}^{-1} m_{D}^{t}
$$

where $M_{R}$ is not diagonalized, to be compared with the seesaw formula (70) in the case of the ESM.

Notice the important point that in Section 1 we have disregarded the possibility in the LRM of a Higgs triplet $\Delta_{L}$ that in principle could also contribute to the mass of the light neutrinos (see for example [3, 26]), so that formula (84) is only correct in the LRM if one neglects this type II seesaw contribution.

Equation (82) implies, using the approximate unitarity of $T$ :

$$
R \simeq m_{D} T^{*}\left(M_{R}^{\text {diag }}\right)^{-1}
$$

to be distinguished from (71), that holds in the ESM case. We see that in the LR case the PMNS matrix $T$ of the heavy neutrinos $T$ enters in the matrix $R$ and, on the other hand, the matrix $S$ satisfies relation (72) that we found in the ESM.

\subsubsection{Summary of the parameter counting in the mass basis}

We have seen that the set of matrices $K, R, S$ and $T$ have together 21(12) parameters. Unlike the case of the ESM, in the LR model we have enough parameter space to accomodate two different PMNS matrices for $K$ and $T$, with $6(3)$ parameters each. Then, $R$ and $S$ can have together extra 9(6) parameters. However, this situation is not compulsory : there can be overlap between the parameters of all the four matrices $K, R, S$ and $T$.

In conclusion, the parameter counting in the physically interesting solution is as follows : 6(3) parameters from the PMNS-like unitary matrix $K+6(3)$ parameters 
from the PMNS-like matrix $T+9(6)$ extra parameters from the complex matrices $R, S+3(0)$ from $M_{R}^{\text {diag }}+3(0)$ from $m_{L}^{\text {diag }}+3(0)$ from $m_{e}^{\text {diag }}=30(12)$ parameters, the same number as in the current basis.

\subsubsection{Possible observables in the Left-Right model}

The gauge bosons $W_{L}$ and $W_{R}$ are mixed in the Left-Right Model :

$$
W_{L}=\cos \zeta W_{1}-\sin \zeta W_{2}, \quad W_{R}=e^{i \omega}\left(\sin \zeta W_{1}+\cos \zeta W_{2}\right)
$$

where $W_{1}$ and $W_{2}$ are mass eigenstates, and the mixing angle $\zeta$, in terms of the vacuum expectation values (12,15), is of the order [15]

$$
\zeta \simeq \pm \frac{g_{L}}{g_{R}} \frac{2\left|k_{1} k_{2}\right|}{\left|v_{R}\right|^{2}}
$$

From (76), it is interesting to write down the lightest mass vector boson $W_{1}$ couplings to leptons

$$
\mathcal{L}_{w}^{W_{1}}=\left[\cos \zeta\left(\overline{\nu_{L}} K^{\dagger}+\overline{\left(N_{R}\right)^{c}} R^{\dagger}\right) \gamma_{\mu} e_{L}+e^{i \omega} \sin \zeta\left(\overline{N_{R}} T^{t}+\overline{\left(\nu_{L}\right)^{c}} S^{t}\right) \gamma_{\mu} e_{R}\right] W_{1}^{\mu}+\text { h.c. }
$$

Besides the $\sim \cos \zeta$ term that describes the processes $\Delta L=0$ (44) and $\Delta L=2$ (45) as in the ESM case, the subleading term $\sim \sin \zeta$ describes the $\Delta L=0$ process

$$
N_{R} \rightarrow e_{R} W_{1}
$$

and another term describing the lepton-number violating decay $\Delta L=2$ of the gauge boson

$$
W_{1} \rightarrow \bar{e}_{R}\left(\nu_{L}\right)^{c}
$$

$\left(L\left(\bar{e}_{R}\right)=L\left(\left(\nu_{L}\right)^{c}\right)=-L\left(e_{R}\right)=-L\left(\nu_{L}\right)\right)$. However, the amplitude for this latter decay is very small, as we will see below.

On the other hand, the heavier vector boson $W_{2}$ couplings to leptons read:

$\mathcal{L}_{w}^{W_{2}}=\left[-\sin \zeta\left(\overline{\nu_{L}} K^{\dagger}+\overline{\left(N_{R}\right)^{c}} R^{\dagger}\right) \gamma_{\mu} e_{L}+e^{i \omega} \cos \zeta\left(\overline{N_{R}} T^{t}+\overline{\left(\nu_{L}\right)^{c}} S^{t}\right) \gamma_{\mu} e_{R}\right] W_{2}^{\mu}+h . c$.

Here, the subleading $\sim \sin \zeta$ term describes the $\Delta L=0$ process

$$
W_{2} \rightarrow \bar{e}_{L} \nu_{L}
$$


and the $\Delta L=2$ transition, assuming the mass of $W_{2}$ heavier that the one of $N_{R}$ :

$$
W_{2} \rightarrow \bar{e}_{L}\left(N_{R}\right)^{c}
$$

On the other hand, the leading $\sim \cos \zeta$ term describes the process $\Delta L=0$

$$
W_{2} \rightarrow \bar{e}_{R} N_{R}
$$

and the $\Delta L=2$ involving light leptons:

$$
W_{2} \rightarrow \bar{e}_{R}\left(\nu_{L}\right)^{c}
$$

Of course, the phenomenological relevance of the $\Delta L=2$ decay involving the $W_{R}$ gauge boson depends on its mass scale.

Concerning the possibility of physics of the LRM at relatively low energies, with observables at LHC scales, one should however take into account that there are severe constraints on such a low energy LRM. This point has been carefully studied in a detailed paper by Deshpande, Gunion, Kayser and Olness [24], who have examined the relevant constraints : structure of the vacuum, limits on flavor-changing neutral currents, etc. The conclusion is that, although such a low energy LRM is not excluded, it is not natural in a straightforward way, and can only be formulated through some degree of fine-tuning.

If one assumes that the mass scale of the LRM is low, it makes sense to look at the LHC for lepton-number violation processes through the search of $p p \rightarrow \ell \ell j j$ topologies, where the two leptons are of the same charge (see for example the recent refs. [20, 21, 22]).

Indeed, using (91) there is the possibility of the $\Delta L=2$ process

$$
W_{2}^{+} \sim W_{R}^{+} \rightarrow e_{R}^{+} N_{R} \rightarrow e_{R}^{+} e_{L}^{+} W_{L}^{-} \rightarrow e_{R}^{+} e_{L}^{+} j j
$$

where $W_{L}^{-}$decays into two hadronic jets, the subscripts in $e_{R}$ and $e_{L}$ mean the couplings to $W_{R}$ and $W_{L}$, and we use the notation $\left(e_{R}\right)^{c}=e_{R}^{+},\left(e_{L}\right)^{c}=e_{L}^{+}$. The decay chain (96) is the very interesting Keung-Senjanovic process proposed long time ago [23] that tests, at the same time, the decay of the gauge boson $W_{R}$ and the Majorana character of the right handed neutrino $N_{R}$.

The PMNS mixing matrix $T$ of the heavy right-handed neutrinos $N_{R}$ controls the decay $W_{R}^{+} \rightarrow e_{R}^{+} N_{R}$. On the other hand, we see from formula (76) that the 
secondary decay $N_{R} \rightarrow e_{L}^{+} W_{L}^{-}$is controlled by the matrix $R \simeq m_{D} T^{*}\left(M_{R}^{\text {diag }}\right)^{-1}$ (cf. (85)). Therefore, this latter decay is controlled by the Dirac mass [23] in the basis in which $M_{R}$ is diagonalized, $m_{D}^{\prime}=m_{D} T^{*}$ (see below the leptogenesis part).

The decay chain (96) through $W_{R}^{+} \rightarrow e_{R}^{+} N_{R} \rightarrow e_{R}^{+} e_{L}^{+} W_{L}^{-}$depends on both matrices $T$ and $R$. Let us suppose that, through the kinematics of the two jets in the decay $W_{L}^{-} \rightarrow j j$, one can reconstruct the $W_{L}^{-}$boson. Then, the angular distribution of the three body decay $W_{R}^{+} \rightarrow e_{R}^{+} e_{L}^{+} W_{L}^{-}$will give information on the matrices $T$ and $R$.

The discussion of the observables in these decay chains depends on the assumed $N_{R}$ spectrum. One usually assumes that the gauge boson $W_{R}$ has a mass bigger or of the order of the heaviest $N_{R}$, that would correspond to a Yukawa coupling of $O(1)$, in analogy with the top quark. However, to simplify what follows, let us assume that all $N_{R_{i}}(i=1,2,3)$ are lighter than the $W_{R}$.

From relation (85) one can see that, in the limit of degenerate heavy neutrinos, summing over the three $N_{R_{i}}$, the amplitude for the process $W_{R}^{+} \rightarrow e_{R}^{+} e_{L}^{+} W_{L}^{-}$depends on the product $m_{D}^{*} T M_{R}^{-1}$, where $M_{R}$ is the non-diagonalized right-handed neutrino mass.

One relevant question is to ask whether one can measure the PMNS mixing matrix $T$. Then, although quite difficult, the $W_{L}$ could in principle be reconstructed through its decays into two jets $W_{L} \rightarrow j j$, and the different $N_{R_{i}}$ could be reconstructed as well through the decays $N_{R_{i}} \rightarrow e_{L}^{+} W_{L}^{-}$.

Our starting point was the mass Lagrangian where the charged lepton part is diagonalized (73), and the final output was the interaction Lagrangian (76)), where the decays $W_{R}^{+} \rightarrow e_{R}^{+} N_{R_{i}}(i=1,2,3)$ depend on the PMNS matrix $T$. Considering the possibility of the three leptons $e_{i}(i=1,2,3)$ of the Standard Model $e, \mu, \tau$, we see that through the rates of these decays, the moduli of all the matrix elements $T_{i j}$ are in principle accessible to experiment.

\section{Representations of the Dirac mass matrix}

The Dirac mass matrix $m_{D}$ is a crucial input in neutrino physics, making the link between high and low energy. We review now some useful representations of $m_{D}$. 


\subsection{Triangular parametrization}

An interesting representation of the Dirac mass matrix $m_{D}$ has been proposed by Branco et al. [10]:

$$
m_{D}=U m_{\Delta}
$$

where $U$ is a unitary matrix with 6(3) parameters of the PMNS form, although not identical to it, and $m_{\Delta}$ is a triangular matrix, with 3 vanishing off-diagonal elements, 3 real diagonal elements and 3 complex off-diagonal elements.

The factorization formula (97) is usually called in Mathematics QR Decomposition of a complex square matrix $M$. In Mathematica notation [25] QRDecomposition[M] gives the decomposition of a numerical complex matrix $M$ in terms of a unitary matrix $U$ and an upper triangular matrix $m_{\Delta}$, while [9, 10] refers to a lower triangular matrix, although this is not an essential point. This decomposition can be numerically very useful for texture models of the matrix $m_{D}$, since it isolates $m_{\Delta}$, and hence the parameters that are relevant for leptogenesis.

The counting of parameters for $m_{D}$ holds in (97) : 15(6) parameters of $m_{D}=$ $6(3)$ parameters of $U+9(3)$ parameters from the triangular matrix $m_{\Delta}$. Relation (97) also holds if $m_{D}$ is general complex and $U$ a general unitary matrix : 18(9) parameters of $m_{D}=9(6)$ parameters of $U+9(3)$ parameters from the triangular matrix $m_{\Delta}$. In the same way that 3 phases of $m_{D}$ can be rotated away by the transformation (24,26), and one can consistently rotate away 3 phases of the general unitary matrix $U$ [10].

Relation (97) is non-trivial. Indeed, because of the unitarity of $U$ we see that $m_{D}^{\dagger} m_{D}$ is given by

$$
m_{D}^{\dagger} m_{D}=m_{\Delta}^{\dagger} m_{\Delta}
$$

and therefore the three CP phases of $m_{\Delta}$ control the amount of leptogenesis at high energies in the one-flavor approximation.

\subsubsection{Extended Standard Model}

With (97), equation (71) obtained within the seesaw, writes

$$
R \simeq U m_{\Delta}\left(M_{R}^{\text {diag }}\right)^{-1}
$$


We have seen above that if we decide that $K$ is of the PMNS type with 6(3) parameters, then the parameters of $K$ have to be chosen among the ones of $R$. A solution satisfying this criterium is a Dirac mass matrix given by [9]

$$
m_{D}=K m_{\Delta}, \quad R \simeq K m_{\Delta}\left(M_{R}^{\text {diag }}\right)^{-1}
$$

Besides its historical interest, this solution has the very nice feature of factorization of the Dirac mass matrix into two pieces, a low energy PMNS mixing matrix $K$ with $6(3)$ parameters, and a high energy mass matrix $m_{\Delta}$, that has $9(3)$ parameters and controls leptogenesis.

Another extreme case would be to assume that $U=1$ [27, 28] that implies

$$
m_{D}=m_{\Delta}, \quad R \simeq m_{\Delta}\left(M_{R}^{\text {diag }}\right)^{-1}
$$

This ansatz relates directly the CP-violating phase in leptogenesis and CP-violation at low energy in neutrino oscillations.

However, there are many other solutions, since in all generality one can choose the parameters of $K$ among the ones of the product $m_{D}=U m_{\Delta}$.

\subsubsection{Left-Right Model}

Equation (85), writes

$$
R=U m_{\Delta} T^{*}\left(M_{R}^{\text {diag }}\right)^{-1}
$$

where we see that the matrix $T$, unlike the case of the ESM (99), enters in the definition of the matrix $R$, that controls leptogenesis.

\subsection{The orthogonal parametrization}

Another useful parametrization of $m_{D}$ has been proposed by Casas and Ibarra [29].

\subsubsection{Extended Standard Model}

Starting from the seesaw formula (70) and diagonalizing $m_{L}$ by the PMNS matrix $K(\underline{69})$,

$$
m_{L}^{\text {diag }}=-K^{\dagger} m_{D}\left(M_{R}^{\text {diag }}\right)^{-1} m_{D}^{t} K^{*}
$$


As pointed out in [29], this relation implies,

$$
-\left(m_{L}^{\text {diag }}\right)^{-1 / 2} K^{\dagger} m_{D}\left(M_{R}^{\text {diag }}\right)^{-1 / 2}\left(M_{R}^{\text {diag }}\right)^{-1 / 2} m_{D}^{t} K^{*}\left(m_{L}^{\text {diag }}\right)^{-1 / 2}=1
$$

and therefore the matrix $i\left(m_{L}^{\text {diag }}\right)^{-1 / 2} K^{\dagger} m_{D}\left(M_{R}^{\text {diag }}\right)^{-1 / 2}$ is an orthogonal complex matrix $O$

$$
O=i\left(m_{L}^{\operatorname{diag}}\right)^{-1 / 2} K^{\dagger} m_{D}\left(M_{R}^{\text {diag }}\right)^{-1 / 2}
$$

i.e. $O O^{t}=1$. One finds the general expression for $m_{D}$ in terms of the matrix $O$

$$
m_{D}=-i K\left(m_{L}^{\text {diag }}\right)^{1 / 2} O\left(M_{R}^{\text {diag }}\right)^{1 / 2}
$$

One can check from this expression that $m_{D}=K m_{\Delta}$ (100) is not the most general form for $m_{D}$ because $O$, being a general complex orthogonal matrix, the combination $-i\left(m_{L}^{\text {diag }}\right)^{1 / 2} O\left(M_{R}^{\text {diag }}\right)^{1 / 2}$ is not triangular in general.

The parametrization (106) is very useful to analyze leptogenesis $C P$ asymmetries when taking flavor into account.

\subsubsection{Left-Right Model}

From eq. (83) one gets, instead of (104)

$$
-\left(m_{L}^{\text {diag }}\right)^{-1 / 2} K^{\dagger} m_{D} T^{*}\left(M_{R}^{\text {diag }}\right)^{-1 / 2}\left(M_{R}^{\text {diag }}\right)^{-1 / 2} T^{\dagger} m_{D}^{t} K^{*}\left(m_{L}^{\text {diag }}\right)^{-1 / 2}=1
$$

that defines the orthogonal matrix

$$
O^{\prime}=i\left(m_{L}^{\text {diag }}\right)^{-1 / 2} K^{\dagger} m_{D} T^{*}\left(M_{R}^{\text {diag }}\right)^{-1 / 2}
$$

and $m_{D}$ is now in the LRM

$$
m_{D}=-i K\left(m_{L}^{\text {diag }}\right)^{1 / 2} O^{\prime}\left(M_{R}^{\text {diag }}\right)^{1 / 2} T^{t}
$$

that includes the PMNS mixing matrix $T$ of right-handed neutrinos.

\subsection{Relation between the triangular and orthogonal forms}

The orthogonal parametrization of the Dirac mass matrix $m_{D}$ appears to be powerful because it explicitly includes low energy quantities, the light neutrino eigenvalues 
$m_{L}^{\text {diag }}$ and the PMNS mixing matrix $K$ and, on the other hand, high energy quantities, the heavy right-handed neutrino eigenvalues $M_{R}^{\text {diag }}$ and an unknown orthogonal complex matrix $O$. One can write down the relation between both representations.

In the ESM, from relation (106) one can write the QR decomposition of the matrix

$$
-i\left(m_{L}^{\text {diag }}\right)^{1 / 2} O\left(M_{R}^{\text {diag }}\right)^{1 / 2}=V m_{\Delta}
$$

where $V$ is another unitary matrix, and $m_{\Delta}$ a triangular matrix. We see therefore that the matrix $m_{D}$ has the form of the triangular parametrization (97) $m_{D}=U m_{\Delta}$, with the PMNS matrix $K$ being a factorizable part of the unitary matrix $U$, namely $U=K V$. Therefore, although one can set $U=1$, i.e. $V=K^{-1}$, and then the low energy phases are part of $m_{\Delta}$ and hence of leptogenesis, the natural solution seems to be that the PMNS matrix $K$ is a unitary factor of the matrix $U$, i.e. $U=K V$, $V$ being a unitary matrix.

\section{$5 \quad$ Leptogenesis}

The gauge models that we consider conserve $B-L$. As nicely pointed out by Strumia [30], the mere existence of sphalerons, that violate $B+L$ in the Standard Model at high temperature, suggests that baryogenesis can proceed via leptogenesis [31, 32]. From (43) or (76), we see that lepton number is violated by the decays of heavy right-handed neutrinos, giving rise to a lepton asymmetry that is partially converted into a baryon asymmetry by the sphalerons. The out-of-equilibrium $\mathrm{CP}$ violating decays of heavy Majorana neutrinos, supplemented by sphaleron interactions, satisfy the three Sakharov criteria [33] to obtain baryogenesis.

In this section we consider leptogenesis in the electroweak broken phase, coming from the $C P$ violating $\Delta L=2$ decay $\left(N_{R}\right)^{c} \rightarrow e_{L} W_{L}$ in the Lagrangians (43) of the ESM and (76) of the LRM.

The actual leptogenesis occurs at very high temperature, in the electroweak unbroken phase. The connection between cosmological $C P$ violation in the unbroken phase [39] with a single massless Higgs doublet, and in the broken phase has been underlined by Branco et al. [10]. In the case of the Left-Right model, this connection is not clear a priori because the massless Higgs fields in the unbroken case belong to 
the bidoublet (10). As we emphasize below, this relation is worth to be investigated. For the moment, we are interested here in the possible differences between the ESM and the LRM in the broken phase, where the interaction Lagrangians (43) and (76) apply.

\subsection{One-flavor approximation}

\subsubsection{Extended Standard Model}

In this part on the ESM we reproduce the results of ref. [10], with the aim of comparing below with the LRM. The lepton number asymmetry from the decay of the 1st, lightest heavy Majorana neutrino, in the broken electroweak phase and in the one-flavor approximation is given by :

$$
\epsilon_{1}=\frac{g^{2}}{M_{W}^{2}} \frac{1}{16 \pi} \frac{1}{\left(R^{\dagger} R\right)_{11}} \sum_{k \neq 1} F\left(x_{k}\right) M_{k}^{2} \operatorname{Im}\left[\left(R^{\dagger} R\right)_{1 k}\right]^{2}
$$

since, from (43), the matrix $R$ is responsible for the transition $\left(N_{R}\right)^{c} \rightarrow e_{L} W_{L}$, or equivalently the decay $\left(N_{R}\right)^{c} \rightarrow e_{L} H$ above the phase transition. In eqn. (111) the function $F\left(x_{k}\right)$ reads

$$
F\left(x_{k}\right)=\sqrt{x_{k}}\left[1+\left(1+x_{k}\right) \ln \left(\frac{x_{k}}{1+x_{k}}\right)+\frac{1}{1-x_{k}}\right] \quad\left(x_{k}=\frac{M_{k}^{2}}{M_{1}^{2}}\right)
$$

As poined out in ref. [10], from (71) $R \simeq m_{D}\left(M_{R}^{\text {diag }}\right)^{-1}$, that holds in the ESM for $m_{D}<<M_{R}$, one gets the lepton number asymmetry in terms of the Dirac mass or, equivalently, in terms of the Yukawa couplings $\frac{m_{D}}{v}$ in the unbroken phase :

$$
\epsilon_{1}=\frac{g^{2}}{M_{W}^{2}} \frac{1}{16 \pi} \frac{1}{\left(m_{D}^{\dagger} m_{D}\right)_{11}} \sum_{k \neq 1} F\left(x_{k}\right) \operatorname{Im}\left[\left(m_{D}^{\dagger} m_{D}\right)_{1 k}\right]^{2}
$$

While the expression of the lepton number asymmetry (111) depends only on quantities of the mass basis, namely on the matrices $R, M_{R}^{\text {diag }}$, expression (113) depends only on quantities of the current basis, since the matrix $M_{R}$ is diagonalized from the beginning in both bases. Notice that, as exposed in [10], expression (113) has a well-defined limit for the SM vacuum expectation value limit $v \rightarrow 0$, given in terms of Yukawa couplings corresponding to the decay in the unbroken electroweak phase $\left(N_{R}\right)^{c} \rightarrow e_{L} H$ [39]. 
In terms of the matrix $m_{\Delta}$ one gets

$$
\epsilon_{1}=\frac{g^{2}}{M_{W}^{2}} \frac{1}{16 \pi} \frac{1}{\left(m_{\Delta}^{\dagger} m_{\Delta}\right)_{11}} \sum_{k \neq 1} F\left(x_{k}\right) \operatorname{Im}\left[\left(m_{\Delta}^{\dagger} m_{\Delta}\right)_{1 k}\right]^{2}
$$

that depends only on the three phases of $m_{\Delta}$.

On the other hand, in terms of the orthogonal matrix $O$ defined in (105) the CP asymmetry is given by

$$
\epsilon_{1}=\frac{g^{2}}{M_{W}^{2}} \frac{1}{16 \pi} \frac{1}{M_{1} \sum_{i} m_{i}\left|O_{i 1}\right|^{2}} \sum_{k \neq 1} F\left(x_{k}\right) M_{1} M_{k} \operatorname{Im}\left[\sum_{j}\left(m_{j} O_{j 1}\right)^{2}\right]
$$

\subsubsection{Left-Right Model}

In the LR model one has in principle two types of contributions to the light neutrino masses, through type I seesaw and type II seesaw, the latter arising from triplet Higgs

exchange (see for example refs. [3, 24, 26]). As pointed out above, in this paper we consider only the contribution of type I seesaw.

In the LR case we have seen that the matrix responsible for the transitions $\left(N_{R}\right)^{c} \rightarrow e_{L} W_{L}$ is the matrix called also $R$ in the mass basis Lagrangian (76). Then, the lepton number asymmetry from the decay of the 1st heavy Majorana neutrino, in the single flavor approximation, is given by the same formulas (111,112).

In the LR model we have now $R$ given by (85), that yields the lepton number asymmetry in terms of the Dirac mass and the mixing matrix $T$ of the heavy neutrinos :

$$
\epsilon_{1}=\frac{g^{2}}{M_{W}^{2}} \frac{1}{16 \pi} \frac{1}{\left(T^{t} m_{D}^{\dagger} m_{D} T^{*}\right)_{11}} \sum_{k \neq 1} F\left(x_{k}\right) \operatorname{Im}\left[\left(T^{t} m_{D}^{\dagger} m_{D} T^{*}\right)_{1 k}\right]^{2}
$$

In the LR model the lepton number asymmetry depends on the current basis matrix $m_{D}$ in (34) and also on the PMNS matrix $T$ of the heavy neutrinos. Consistently, the presence of the matrix $T$ appears in (116) because, to compute the decay rates $\left(N_{1}\right)^{c} \rightarrow e_{L} W_{L}$, one needs first to diagonalize the mass matrix $M_{R}=t v_{R}(15)$.

In other terms, the matrix $m_{D} T^{*}=m_{D}^{\prime}$ is the Dirac mass matrix in the basis in which $M_{R}$ in (27) is diagonalized. In this basis the left-handed term of the interaction Lagrangian $\bar{\nu}_{L} e_{L} W_{L}$ remains diagonal, but the right-handed term $\bar{N}_{R} e_{R} W_{R}$ is not anymore.

Expression (116) for the CP asymmetry in the electroweak broken phase follows from the $R$-term in the interaction Lagrangian (76), responsible for the decay 
$\left(N_{R}\right)^{c} \rightarrow e_{L} W_{L}$. This is the expression that has been used precisely to compute the leptogenesis CP asymmetry within LRM (see for example refs. [26, 40]).

However, in the LRM the broken electroweak phase is more involved than in the ESM because there are two vacuum expectation values $k_{1}$ and $k_{2}$ (12) that contribute to $m_{D}$ and to $M_{W}$, besides the possibility of a vacuum expectation value $v_{L}$ (not considered in subsection 1.1.2) that could also contribute to the $W_{L}$ mass.

In the unbroken electroweak phase, the Higgs bidoublet (10) would be massless, and one should consider both contributions $N_{1} \rightarrow e \varphi_{1,2}$ to the leptogenesis asymmetry, with both Higgses $\varphi_{1,2}$ contributing to the loops needed to interfere with the tree diagram to obtain $C P$ violation. This situation reminds the one of the Standard Model with several Higgs doublets [41]. The relation between the $C P$ asymmetries in the broken and unbroken phases of the LRM deserves further investigation.

Since the matrix $m_{D}$ is general complex, so is $m_{D} T^{*}$ and we can write a decomposition in terms of another general unitary matrix $U^{\prime}$ and another triangular matrix $m_{\Delta}^{\prime}$ :

$$
m_{D}^{\prime}=m_{D} T^{*}=U^{\prime} m_{\Delta}^{\prime}
$$

The lepton asymmetry writes

$$
\epsilon_{1}=\frac{g^{2}}{M_{W}^{2}} \frac{1}{16 \pi} \frac{1}{\left(m_{\Delta}^{\prime \dagger} m_{\Delta}^{\prime}\right)_{11}} \sum_{k \neq 1} F\left(x_{k}\right) \operatorname{Im}\left[\left(m_{\Delta}^{\prime \dagger} m_{\Delta}^{\prime}\right)_{1 k}\right]^{2}
$$

that now depends on the three CP phases of $m_{\Delta}^{\prime}$.

On the other hand, notice that the interaction Lagrangian (76) contains also the $\Delta L=2$ term $\overline{\left(\nu_{L}\right)^{c}} S^{t} e_{R} W_{R}$ that could give a contribution to the lepton asymmetry through the decay

$$
W_{R} \rightarrow \bar{e}_{R}\left(\nu_{L}\right)^{c}
$$

The masses $M_{W_{R}}$ and $M_{i}$ are both generated by the same Higgs triplet, and since one usually assumes that the Yukawa coupling of the heaviest neutrino $N_{3}$ is of $O(1)$, then $M_{W_{R}}>>M_{1}$ assuming a hierarchical spectrum for the heavy neutrinos. Hence, the lepton asymmetry generated by the decay of $W_{R}$ could be washed out and only the one due to the $N_{1}$ decays would survive. However, one should keep in mind in model building the possibility of leptogenesis through the decay (119). 


\section{$5.2 \quad$ Flavored leptogenesis}

\subsubsection{Extended Standard Model}

A crucial progress in leptogenesis has been achieved by taking into account flavor [34, 35, 36, 37. At high temperatures $T \geq 10^{12} \mathrm{GeV}$, all three $\tau, \mu$ and $e$ are out of equilibrium because their Yukawa couplings are weak compared to the temperature. In this regime, the one-flavor approximation can be applied since the different lepton flavors are undistinguishable.

However, for "realistic" temperatures $T \simeq M_{1}$ such that $10^{9} \leq T \leq 10^{12} \mathrm{GeV}$, the $\tau$ lepton doublet Yukawa coupling is large enough to be in termal equilibrium, while the $\mu$ and $e$ doublets are out of equilibrium. The net result is that the leptogenesis $\mathrm{CP}$ violation splits into two pieces, $\epsilon_{\tau}$ and $\epsilon_{2}=\epsilon_{\mu}+\epsilon_{e}$, since the flavors $\mu$ and $e$ remain undistinguishable. Then, in the range $10^{9} \leq T \leq 10^{12} \mathrm{GeV}$, the final baryon asymmetry $Y_{B}$ is the sum of two contributions, given by the lepton $\mathrm{CP}$ asymmetries $\epsilon_{\tau}$ and $\epsilon_{2}$ affected by different wash-out factors $\eta_{\tau}$ and $\eta_{2}: Y_{B} \propto \epsilon_{\tau} \eta_{\tau}+\epsilon_{2} \eta_{2}$. A recent updated flavor covariant description of flavor effects in leptogenesis can be found in ref. [38].

The $\mathrm{CP}$ violating asymmetry for each flavour is given by the expression (see for example [5]) :

$$
\begin{aligned}
\epsilon_{1 \ell} & =\frac{g^{2}}{M_{W}^{2}} \frac{1}{16 \pi} \frac{1}{\left(m_{D}^{\dagger} m_{D}\right)_{11}} \sum_{k \neq 1} F\left(x_{k}\right) \operatorname{Im}\left[\left(m_{D}^{\dagger}\right)_{1 \ell}\left(m_{D}\right)_{\ell k}\left(m_{D}^{\dagger} m_{D}\right)_{1 k}\right]^{2} \\
& +\frac{g^{2}}{M_{W}^{2}} \frac{1}{16 \pi} \frac{1}{\left(m_{D}^{\dagger} m_{D}\right)_{11}} \sum_{k \neq 1} G\left(x_{k}\right) \operatorname{Im}\left[\left(m_{D}^{\dagger}\right)_{1 \ell}\left(m_{D}\right)_{\ell k}\left(m_{D}^{\dagger} m_{D}\right)_{k 1}\right]^{2}
\end{aligned}
$$

where the second term corresponds to the lepton flavor violating but lepton number conserving self-energy diagram [36]. The function $F\left(x_{k}\right)$ is given by (112), and

$$
G\left(x_{k}\right)=\frac{1}{1-x_{k}}
$$

The second term in (120) vanishes when summing over $\ell$, while the first term gives the one-flavor approximation expression (113), because $\sum_{\ell} \epsilon_{1 \ell}=\epsilon_{1}$. On the other hand, the second term in (120) is subleading if one assumes $M_{1}<<M_{2}, M_{3}$.

The flavored wash-out factors read [37]

$$
\eta_{\ell}=\eta \frac{\left(m_{D}^{\dagger}\right)_{1 \ell} m_{D \ell 1}}{\left(m_{D}^{\dagger} m_{D}\right)_{11}}
$$


where $\eta$ is the wash-out factor in the single flavor approximation.

Concerning the link between low energy CP violation in the PMNS mixing matrix and leptogenesis CP violation, the situation is quite different if flavor is taken into account [37]. As an illustration, let us write the $\mathrm{CP}$ asymmetry $\epsilon_{1 \ell}$, where the subindex 1 means decay of the lightest heavy Majorana neutrino $N_{1}$, by using the orthogonal parametrization (106). The flavor CP asymmetries $\epsilon_{1 \ell}$ depend then on the low energy parameters, i.e. the light neutrino masses and the PMNS mixing matrix $K$. Assuming $M_{1}<<M_{2}<M_{3}$, one finds from (106) and (120) the leptonic CP violation parameter $\epsilon_{1 \ell}$ [37] :

$$
\epsilon_{1 \ell} \simeq-\frac{3}{32 \pi} \frac{g^{2}}{M_{W}^{2}} \frac{\operatorname{Im}\left(\sum_{k, j} m_{j} m_{k}^{3 / 2} K_{\ell j}^{*} K_{\ell k} O_{j 1}^{*} O_{k 1}^{*}\right)}{\sum_{i} m_{i}\left|O_{i 1}\right|^{2}}
$$

\subsubsection{Left-Right Model}

As we have seen in the LRM in the one-flavor approximation (formula (116)), $m_{D}$ is replaced by $m_{D} T^{*}$, and the formula for the lepton asymmetry in this approximation is the same as in the Extended Standard model with the replacement $m_{D} \rightarrow m_{D}^{\prime}=$ $m_{D} T^{*}$ where $m_{D}^{\prime}$ is the Dirac mass matrix in the basis in which the mass matrix $M_{R}$ is diagonalized.

Because of (108), formulas for the CP asymmetry (120) and the wash-out factor (122) remain correct for the Left-Right model, with the replacement $m_{D} \rightarrow m_{D}^{\prime}=$ $m_{D} T^{*}$, where $m_{D}$ is given by (109), that has a complete left-right symmetry in the dependence on the mass eigenvalues $m_{L}^{\text {diag }}, M_{R}^{\text {diag }}$ as well as on the mixing matrices $K, T$. Then, the flavor asymmetry has the same form (123), with the replacement $O \rightarrow O^{\prime}$

\section{Comparison between the Extended Standard Model and the Left-Right Model}

We now summarize the comparison between the ESM and the LRM, as far as lepton mixing is concerned.

(a) In the current basis both models differ in the following way. 
In the ESM the Dirac matrix $m_{D}$ has 15(6) parameters because one can rotated away 3 phases and one can diagonalize the right-handed mass matrix $M_{R}$. One has finally a total of 21(6) parameters.

In the LRM one cannot diagonalize $M_{R}$ without changing the interaction Lagrangian. On the other hand, one cannot rotate away phases in both $m_{D}$ and in $M_{R}$, but only three phases in one of these matrices, that we have chosen to be $M_{R}$. Then, one is left with a general complex $m_{D}$ with 18(9) parameters and a complex symmetric $M_{R}$ with $9(3)$ parameters. With the $m_{e}$ mass eigenvalues, this gives a total of 30(12) parameters.

However, if in the LRM one diagonalizes $M_{R}$ from the start, the left-handed interaction term $\bar{\nu}_{L} \gamma_{\mu} e_{L} W_{L}^{\mu}$ remains diagonal, while the right-handed term $\bar{N}_{R} \gamma_{\mu} e_{R} W_{R}^{\mu}$ is modified. Also $m_{D}$ is modified to another Dirac mass term, that would eventually control leptogenesis. Therefore, as far as one considers the mass terms and the $W_{L}$ interation, one has the same number of parameters as in the ESM. For physics at low energy and also for leptogenesis, if the latter is attributed to the decays of the lightest right-handed heavy neutrino $N_{1}$, one can disregard the $W_{R}$ interaction term, that involves heavier degrees of freedom.

(b) In the mass basis in the ESM without approximations one has two mixing matrices $K$ and $R$ in the left sector, that have together 12(6) parameters. For $m_{D}<<M_{R}$ one has a priori 12(6) parameters for the set of matrices $K, R$ (mixing in the left sector), and $S, T$ (mixing in the right sector). The mixing matrix of the left-handed neutrinos is approximately unitary and can be chosen to be of the PMNS type, with 6(3) parameters. The model constrains the mixing matrix of the righthanded neutrinos to be $T \simeq 1$, the matrix $R$ (71) has a total of to 12(6) parameters and $S$ is not independent because of relation (72). The parameters of the PMNS mixing matrix for light neutrinos $K$ have to chosen among the ones of $R$. Adding the mass eigenvalues $m_{L}^{\text {diag }}, M_{R}^{\text {diag }}, m_{e}^{\text {diag }}$ one has a total of 21(6) parameters

In the LRM in the mass basis one has more symmetry : two mixing matrices $K, R$ in the left sector and two $S, T$ in the right sector. These four matrices have together 21(12) parameters, that added to the mass eigenvalues $m_{L}^{\text {diag }}, M_{R}^{\text {diag }}, m_{e}^{\text {diag }}$ gives again a total of 30(12) parameters. In the approximation $m_{D}<<M_{R}$ the mixing matrices $K$ (left sector) and $T$ (right sector) are unitary, and both can be 
chosen to be of the PMNS type, with 6(3) parameters each. This is different from the ESM for the right sector, where $T$ is trivial. This feature of the ESM seems unnatural, since physically one should expect a full PMNS matrix for the heavy right-handed neutrinos as well.

(c) Adopting the decomposition $m_{D}=U m_{\Delta}$ ( $U$ unitary and $m_{\Delta}$ triangular complex), in the ESM the matrix $U$ has $6(3)$ parameters and $m_{\Delta} 9(3)$ parameters, corresponding to the 15(6) parameters of $m_{D}$. The natural solution is that the PMNS matrix $K$ is a unitary factor of the matrix $U$, namely $U=K V, V$ being also unitary. In the LRM the situation is somewhat different : $m_{D}$ is a general complex matrix with $18(9)$ parameters, $U$ is a general unitary matrix with $9(6)$ parameters and $m_{\Delta}$ has also $9(3)$ parameters. The Dirac mass matrix in the basis in which $M_{R}$ is diagonal (117) $m_{D}^{\prime}=m_{D} T^{*}$ can be decomposed in the same way : $m_{D}^{\prime}=U^{\prime} m_{\Delta}^{\prime}$.

(d) Concerning the lepton asymmetry relevant for leptogenesis, we find the following situation in both models.

In the ESM, in the one-flavor approximation, the asymmetry is dependent on matrix elements of the matrices $R^{\dagger} R$ or $m_{D}^{\dagger} m_{D}$ or $m_{\Delta}^{\dagger} m_{\Delta}$, i.e. dependent on the 3 $\mathrm{CP}$ phases of $m_{\Delta}$. In the flavored case, the asymmetry (120) depends on the PMNS matrix $K$ and the three high energy phases of the orthogonal matrix $O(105)$.

In the LRM, in the one-flavor approximation, the lepton asymmetry is dependent on $R^{\dagger} R$ or $T^{t} m_{D}^{\dagger} m_{D} T^{*}$. Writing the product $m_{D} T^{*}$ as in (117), the asymmetry depends on the three CP phases of the triangular matrix $m_{\Delta}^{\prime}$ through $m_{\Delta}^{\prime \dagger} m_{\Delta}^{\prime}$. In the flavored case, the asymmetry depends on the three phases of the PMNS mixing matrix $K$ and on the three phases of $O^{\prime}(108)$.

As far as model building is concerned, the situation is different in both schemes. As an example, imagine that one has a model for the Yukawas with some ansatz for $m_{D}$ and $M_{R}$. In the ESM, $M_{R}$ is diagonalized and $m_{D}$ is enough to compute the lepton asymmetry. In the LRM one needs to compute the matrix $T$ that diagonalizes $M_{R}$, in order to get $m_{D}^{\prime}$.

(e) A possible identification between low energy phases and leptogenesis phases is not possible in general. In the ESM one could imagine models in which the three $\mathrm{CP}$ phases of the light neutrinos mixing matrix $K$ are the same as the three phases 
of the triangular matrix $m_{\Delta}$, since one has to choose the parameters of $K$ among the ones of the matrix $R$ in the lepton asymmetry formula (111). In the LRM one could choose the three phases of $K$ to be the same as the ones of $m_{\Delta}^{\prime}$ (117).

As to whether in general the leptogenesis CP asymmetry could depend on the low energy phases, in the flavored regime the usual argument that $\epsilon_{1 \ell}$ in the ESM depend on the PMNS matrix $K$ and on the matrix $O$ (105) extends to the LRM with another orthogonal matrix $O^{\prime}$ (108).

(f) Relatively to the ESM, we have found that the LRM has some interesting new features :

- The non-trivial PMNS mixing matrix $T$ of the heavy neutrinos enters in the quantitative estimation of decay branching ratios of heavy neutrinos $N_{R_{i}}$ to various final states.

- On the other hand, in the calculation of the leptogenesis CP asymmetries, the matrix $T$ is unobservable because the Dirac matrix that plays a role is now (117) $m_{D}^{\prime}=m_{D} T^{*}$, the Dirac matrix in the basis in which $M_{R}$ is diagonal.

- The term $\overline{\left(\nu_{L}\right)^{c}} S^{t} e_{R} W_{R}$ in (176) could give a contribution to the cosmological lepton asymmetry through the $\Delta L=2$ lepton number violating decay to light leptons $W_{R} \rightarrow \bar{e}_{R}\left(\nu_{L}\right)^{c}$. As we have indicated above, this latter possibility seems unlikely in reasonable left-right models because $W_{R}$ is heavier than the lightest neutrino $N_{1}$. However, one should keep in mind this possibility in model building.

- Considering the $W_{1}, W_{2}$ basis, i.e. without neglecting $W_{L}-W_{R}$ mixing, we have seen in Section 3.2 that there is a term involving the lighter $W_{1}$ boson $\sim$ $\sin \zeta \overline{\left(\nu_{L}\right)^{c}} S^{t} \gamma_{\mu} e_{R} W_{1}^{\mu}$, that allows for the subleading $\Delta L=2$ lepton-number violating decay to light leptons $W_{1} \rightarrow \bar{e}_{R}\left(\nu_{L}\right)^{c}$.

\section{Extension to Pati-Salam and $S O(10)$}

One can extend the precedent considerations to other left-right gauge models like the Pati-Salam gauge theory $S U(4)_{C} \times S U(2)_{L} \times S U(2)_{R}$ [17] or $S O(10)$ [6].

We can consider first each of these models in the current basis, with general mass terms determined only by the Dirac or Majorana character of the fermions, and perform the counting of the $C P$ conserving and $C P$ violating free parameters. 
In a second step, one can diagonalize the mass matrices and obtain mixing in the interaction terms and, in a third step, switch on the Higgs sector of each theory and see how, according to the different hypothesis on this sector, the predictive power of each scheme is improved. Of course, with the most general Higgs structure for each model, one populates the general parameter space of the mass terms obtained by imposing only Lorentz invariance.

Moreover, since in these theories leptons are related to quarks, lepton mixing in the Dirac mass term will be related to quark mixing, at least for some Higgs structures. This feature is interesting in view of increasing the predictive power of $S O(10)$ for leptogenesis, and has been used more or less quantitatively in the literature.

Let us give some details for the Pati-Salam model and for $S O(10)$. Consider first the general mass Lagrangian consistent with Lorentz invariance of Dirac and Majorana mass terms

$$
\mathcal{L}_{m}=\bar{\nu}_{L} m_{D} N_{R}+\frac{1}{2} \overline{\left(N_{R}\right)^{c}} M_{R} N_{R}+\bar{e}_{L} m_{e} e_{R}+\bar{u}_{L} m_{u} u_{R}+\bar{d}_{L} m_{d} d_{R}+\text { h.c. }
$$

For the moment the matrices $m_{D}, m_{e}, m_{u}$ and $m_{d}$ are general complex with 18(9) parameters each and $M_{R}$ is a general complex symmetric matrix with 12(6) parameters. This gives a priori a total of $84(42)$ parameters, while in the lepton sector one has 18(9) (from $\left.m_{D}\right)+18(9)\left(\right.$ from $\left.m_{e}\right)+12(6)\left(\right.$ from $\left.M_{R}\right)=48(24)$ parameters.

In the Pati-Salam model and in $S O(10)$, the interaction Lagrangian has the general form

$$
\mathcal{L}_{\text {int }}=\mathcal{L}_{w}+\mathcal{L}_{x}
$$

where one has in both models, keeping only the interesting flavor-changing terms :

$$
\mathcal{L}_{w}=\bar{e}_{L} \gamma_{\mu} \nu_{L} W_{L}^{\mu}+\bar{e}_{R} \gamma_{\mu} N_{R} W_{R}^{\mu}+\bar{d}_{L} \gamma_{\mu} u_{L} W_{L}^{\mu}+\bar{d}_{R} \gamma_{\mu} u_{R} W_{R}^{\mu}+\text { h.c. }
$$

The extra interaction term writes in the Pati-Salam model :

$$
\mathcal{L}_{x}^{P S}=\bar{e}_{L} \gamma_{\mu} d_{L} X_{L}^{\mu}+\bar{e}_{R} \gamma_{\mu} d_{R} X_{R}^{\mu}+\bar{\nu}_{L} \gamma_{\mu} u_{L} X_{L}^{\mu}+\bar{N}_{R} \gamma_{\mu} u_{R} X_{R}^{\mu}+\text { h.c. }
$$

where the colored gauge bosons have charges $\left|Q\left(X_{L}\right)\right|=\left|Q\left(X_{R}\right)\right|=\frac{2}{3}$. 
In $S O(10)$ one has [42, 43] :

$$
\begin{aligned}
\mathcal{L}_{x}^{S O(10)} & =\left[\epsilon^{i j k} \overline{\left(u_{R}^{i}\right)^{c}} \gamma_{\mu} u_{L}^{j}+\overline{d_{L}^{k}} \gamma_{\mu}\left(e_{R}\right)^{c}-\overline{e_{L}} \gamma_{\mu}\left(d_{R}^{k}\right)^{c}\right] X^{k \mu} \\
& +\left[\epsilon^{i j k} \overline{\left(u_{R}^{i}\right)^{c}} \gamma_{\mu} d_{L}^{j}+\overline{\nu_{L}} \gamma_{\mu}\left(d_{R}^{k}\right)^{c}-\overline{u_{L}^{k}} \gamma_{\mu}\left(e_{R}\right)^{c}\right] Y^{k \mu} \\
& +\left[\epsilon^{i j k} \overline{\left(d_{R}^{i}\right)^{c}} \gamma_{\mu} u_{L}^{j}+\overline{e_{L}} \gamma_{\mu}\left(u_{R}^{k}\right)^{c}-\overline{d_{L}^{k}} \gamma_{\mu}\left(N_{R}\right)^{c}\right] Y^{\prime k \mu} \\
& +\left[\epsilon^{i j k} \overline{\left(d_{R}^{i}\right)^{c}} \gamma_{\mu} d_{L}^{j}+\overline{\nu_{L}} \gamma_{\mu}\left(u_{R}^{k}\right)^{c}-\overline{u_{L}^{k}} \gamma_{\mu}\left(N_{R}\right)^{c}\right] X_{D}^{k \mu} \\
& +\left[\overline{\nu_{L}} \gamma_{\mu} u_{L}^{k}+\overline{e_{L}} \gamma_{\mu} d_{L}^{k}-\overline{\left(d_{R}^{k}\right)^{c}} \gamma_{\mu}\left(e_{R}\right)^{c}-\overline{\left(u_{R}^{k}\right)^{c}} \gamma_{\mu}\left(N_{R}\right)^{c}\right] S^{k \mu}+h . c .
\end{aligned}
$$

where $i, j, k$ are color indices and the colored gauge bosons $X, Y, Y^{\prime}, X_{D}, S$ have the charges : $|Q(X)|=\frac{4}{3},|Q(Y)|=\left|Q\left(Y^{\prime}\right)\right|=\frac{1}{3},\left|Q\left(X_{D}\right)\right|=|Q(S)|=\frac{2}{3}$.

Let us see how many parameters can be rotated away in both models. Analogously to the LRM, one can diagonalize $m_{e}$ and absorb 3 phases in $M_{R}$ in (124) while keeping $\mathcal{L}_{w}$ (126) invariant. However, as it is obvious from (127]128), $\mathcal{L}_{x}$ is changed under these transformations. In the pure lepton sector, leaving aside the quark-lepton terms in $\mathcal{L}_{x}$, the starting point for the diagonalization of the mass terms is the same as in the LRM (34), with 30(12) parameters in $m_{D}, M_{R}$ and $m_{e}^{\text {diag }}$. Diagonalizing (124) one gets the flavor-changing mixing in the interaction Lagrangian $\mathcal{L}_{w}+\mathcal{L}_{x}$.

In the pure lepton sector our conclusions are the following. The diagonalization has the same form for $S U(2)_{L} \times S U(2)_{R} \times U(1)$, Pati-Salam and $S O(10)$ models. Separately, the $3 \times 3$ matrices $K$ and $R$ enter in the left sector, while the $3 \times 3$ matrices $T$ and $S$ enter in the right sector, like in the LRM, eqn. (76). In $S U(2)_{L} \times$ $S U(2)_{R} \times U(1)$, Pati-Salam and $S O(10)$ models we have in the lepton sector the same counting of free parameters, i.e. 30 real parameters, among them $12 \mathrm{CP}$-violating phases.

Let us now make some remarks on masses and mixing in some particular cases in the interesting $S O(10)$ case. Let us look at the product

$$
16 \times 16=10_{S}+\overline{126}_{S}+120_{A}
$$

where $\mathbf{1 0}+\overline{\mathbf{1 2 6}}$ is the symmetric part and $\mathbf{1 2 0}$ the antisymmetric part. The representatios $\mathbf{1 0}$ and $\mathbf{1 2 0}$ are real, $\mathbf{1 2 6}$ is complex, and the Yukawa terms that can give mass to the fermions are

$$
16_{f} \times 16_{f} \times 10_{H}=1+\ldots
$$




$$
\begin{aligned}
& 16_{f} \times 16_{f} \times \overline{126}_{H}=1+\ldots \\
& 16_{f} \times 16_{f} \times 120_{H}=1+\ldots
\end{aligned}
$$

The Yukawa part of the Lagrangian reads

$$
\mathcal{L}_{Y}=\mathbf{1 6}_{f}\left(Y_{10} \mathbf{1 0}_{H}+Y_{126} \overline{\mathbf{1 2 6}}_{H}+Y_{120} \mathbf{1 2 0}_{H}\right) \mathbf{1 6}_{f}
$$

where a possible sum over Higgs representations and Yukawa coupling matrices in family space is implicit. After spontaneous symmetry breaking one gets the mass Lagrangian (see for example [44])

$$
\begin{aligned}
& m_{d}=v_{10}^{d} Y_{10}+v_{126}^{d} Y_{126}+v_{120}^{d} Y_{120} \\
& m_{u}=v_{10}^{u} Y_{10}+v_{126}^{u} Y_{126}+v_{120}^{u} Y_{120} \\
& m_{e}=v_{10}^{d} Y_{10}-3 v_{126}^{d} Y_{126}+v_{120}^{e} Y_{120} \\
& m_{D}=v_{10}^{u} Y_{10}-3 v_{126}^{u} Y_{126}+v_{120}^{D} Y_{120} \\
& M_{R}=v_{126}^{R} Y_{126}
\end{aligned}
$$

where the Yukawa matrices $Y_{10}$ and $Y_{126}$ are complex symmetric, $Y_{120}$ is complex antisymmetric, and the $v$ 's are Higgs vacuum expectation values. From the term (130) alone we obtain the well-known relations $m_{e}=m_{d}$ and $m_{D}=m_{u}$, while the term (131) alone would give the relations $m_{e}=-3 m_{d}$ and $m_{D}=-3 m_{u}$, and $n o$ relation from the term (132).

The vev's in (134) are in all generality complex numbers if we assume that $C P$ can be spontaneously broken (soft $C P$ violation). If $C P$ is not spontaneously broken the vevs are real and all $C P$ violation comes from the Yukawa couplings (hard $C P$ violation).

One could wonder how within $\mathrm{SO}(10)$ one can get the most general counting of parameters done above, i.e. 84(42) parameters for the whole mass sector (124), with 48(24) parameters in the lepton sector. As said above, this is simply achieved if all the representations $\mathbf{1 0}_{H}, \overline{\mathbf{1 2 6}}_{H}, \mathbf{1 2 0}_{H}$ in (134) are present and are different for each mass matrix, that becomes then completely general.

An interesting particular case is to consider only the $\mathbf{1 0}$ and $\overline{\mathbf{1 2 6}}$ representations in (134), with 120 absent :

$$
m_{d}=m_{10}^{d}+m_{126}^{d}
$$




$$
\begin{aligned}
& m_{u}=m_{10}^{u}+m_{126}^{u} \\
& m_{e}=m_{10}^{d}-3 m_{126}^{d} \\
& m_{D}=m_{10}^{u}-3 m_{126}^{u} \\
& M_{R}=m_{126}^{R}
\end{aligned}
$$

In this situation, all mass matrices $m_{u}, m_{d}, m_{D}, m_{e}$ and $M_{R}$ are complex symmetric.

Let us count again the number of parameters under this hypothesis. The complex symmetric matrices $m_{10}^{d}, m_{126}^{d}, m_{10}^{u}, m_{126}^{u}, m_{126}^{R}$, have 12(6) parameters each, that gives a total number of 60(30) parameters, a reduction relatively to the $84(42)$ total number of parameters of the general case. One can diagonalize the complex symmetric matrices $m_{d}, \ldots M_{R}$ with unitary matrices $V_{d}, \ldots V_{R}$. Because of relations (135), the unitary matrices $V_{e}, V_{D}, V_{R}$ are in principle given in terms of $V_{u}$ and $V_{d}$ and mass eigenvalues. Notice that, as discussed in the mass basis for the pure lepton sector, we can adopt without loss of generality the basis in which $m_{e}=m_{e}^{\text {diag }}$. However, these relations give complicated equations between the elements of mixing matrices. Within this case of considering both $\mathbf{1 0}$ and $\overline{\mathbf{1 2 6}}$, it seems hard to find relations between the mixing matrices in the quark and the lepton sector, at least in a model-independent way.

Let us consider two limiting cases: while the $\overline{\mathbf{1 2 6}}$ contributes to $M_{R}$, only the $\mathbf{1 0}$ or only the $\overline{\mathbf{1 2 6}}$ contribute to $m_{d}, m_{u}, m_{e}$ and $m_{D}$.

From (135) we see that in both cases one has quark-lepton symmetry in the mixing matrices, i.e. a relation between the left-handed neutrino Dirac mixing matrix $V_{L}$, where $m_{D}=V_{L}^{\dagger} m_{D}^{\operatorname{diag}} V_{R}$, and the CKM quark matrix

$$
V_{L}=V_{u} V_{d}^{\dagger}=V_{C K M}
$$

This relation has been often used in a number of phenomenological schemes [2, 4, 5]. However, as it is well known, one needs both representations $\mathbf{1 0}$ and $\overline{\mathbf{1 2 6}}$ to describe fermion masses in $S O(10)$ [45, 46], and therefore we must conclude that there is a clash between a good description of fermion masses and the one of obtaining quarklepton symmetry in mixing.

Although the point of view of obtaining useful theoretical hints from $S O(10)$ on the eigenvalues and mixing of the Dirac neutrino mass matrix has been advanced 
in a number of works [1, 2, 3, 4, 5], it is worth to point out that there could be an alternative philosophy concerning the Dirac mass matrix. Within the Left-Right Model, if the $W_{R}$ gauge boson and the lightest heavy neutrino $N_{R}$ are light enough, there is the interesting possibility of a complete determination of the Dirac mass matrix from the experimental study of $W_{R}$ and $N_{R}$ decays [47].

\section{Conclusions}

We have examined the parameter counting and structure of CP conserving and CP violating lepton mixing in two gauge models in the electroweak broken phase, the Extended Standard Model - i.e. the Standard Model plus one right-handed heavy neutrino per generation -, and the Left-Right Model $S U(2)_{L} \times S U(2)_{R} \times U_{B-L}(1)$. We have used both the "current basis", in which the gauge interactions are diagonal, and the "mass basis", where the mass matrices are diagonal and mixing appears in the charged current gauge-fermion part of the Lagrangian. On the other hand, we have distinguished between results that are exact and results that hold within the approximation of Dirac masses that are small relatively to right-handed neutrino masses, $m_{D}<<M_{R}$.

We think that it is worth to compare these two models. One reason is that, for simplicity, in the literature people usually discuss lepton mixing within the simple ESM, while actually have in mind left-right Grand Unified Theories like $S O(10)$, that naturally include heavy right-handed neutrinos. The simplest LR model that we study in this paper is a kind of prototype for these more involved LR theories.

Although the outline of the parameter counting and structure of lepton mixing is rather close in both schemes, there are differences between the two models. In particular, the Extended Standard Model can accomodate a PMNS mixing matrix $K$ for light neutrinos, but there is no room in parameter space for a mixing matrix $T$ for the heavy neutrinos, the mixing matrix being close to the identity. On the other hand, as one could naturally expect, the Left-Right Model is consistent with PMNS mixing matrices for both light and heavy neutrinos. The lepton asymmetry relevant for leptogenesis depends, not only on the Dirac mass $m_{D}$, but also on the matrix $T$, that is non-trivial. But the lepton asymmetry is given in terms of the Dirac mass in 
the basis in which the right-handed heavy neutrino mass matrix is diagonal, while the interaction term in the right-handed sector is not diagonal anymore.

In the case of the LR model, the connection between the lepton $C P$ asymmetry in the electroweak broken phase, coming from the decay $\left(N_{R}\right)^{c} \rightarrow W_{L} e_{L}$ and its $C P$ conjugate, and the one in the unbroken phase coming from the decay above the phase transition $N_{R} \rightarrow e \varphi$, where $\varphi$ is the Higgs bidoublet, is an open problem worth to be investigated.

Mixing in the LRM contains new terms that involve $\Delta L=2 \mathrm{CP}$ violating interactions involving the $W_{R}$ gauge bosons. Considering the $W_{L}-W_{R}$ mixing, there are interesting new possible $\Delta L=2$ processes with light leptons in the final state : the subleading decay $W_{1} \rightarrow \bar{e}_{R}\left(\nu_{L}\right)^{c}$ and the leading one $W_{2} \rightarrow \bar{e}_{R}\left(\nu_{L}\right)^{c}$. As emphasized above, it is worth to keep in mind, in model building, the possibility of the latter as a contribution to leptogenesis.

We have extended these results to other LR theories, namely the Pati-Salam model $S U(4)_{C} \times S U(2)_{L} \times S U(2)_{R}$ and the grand unified model $\mathrm{SO}(10)$, for which we find that the structure of mixing in the lepton sector is, in the most general case, the same as in the Left-Right Model $S U(2)_{L} \times S U(2)_{R} \times U_{B-L}(1)$. The specification of the Higgs sector provides schemes that have more predictive power.

If one assumes both symmetric 10 and 126 Higgs representations, necessary to describe the quark mass spectrum, we emphasize that there is a clash between the description of this spectrum and the assumption that the left-handed Dirac mixing matrix is approximately given by the quark CKM matrix, as sometimes it is assumed in phenomenological models arguing naive quark-lepton symmetry.

Phenomenological analyses are usually done within these gauge models as $S O(10)$ supplemented by simplifying hypotheses that give tractable schemes. But one should keep in mind that the general parameter space can yield other possibilities concerning the description of the interesting observables.

Concerning low energy observables, there are no differences between the Extended Standard model and the minimal Left-Right model at leading order in $m_{D} / M_{R}$. The cosmological baryon asymmetry via leptogenesis above the electroweak phase transition deserves however further investigation within the LeftRight model. 


\section{Appendix}

\section{A general digression on the matrices $K, R, S, T$}

To count the number of independent parameters in each scheme, it is useful to consider the general case of diagonalization of a $6 \times 6$ complex symmetric matrix,

$$
\mathcal{M}=\left(\begin{array}{ll}
m_{L} & m_{D} \\
m_{D}^{t} & M_{R}
\end{array}\right)
$$

where $m_{L}$ and $M_{R}$ are $3 \times 3$ complex symmetric. In general, a $6 \times 6$ complex symmetric matrix has $42(21)$ real parameters.

Let us now diagonalize $\mathcal{M}$ with the unitary matrix $V$ (37.399). The unitarity condition $V V^{\dagger}=1$ is an hermitian relation that implies 36(15) constraints. A general complex $6 \times 6$ matrix has $72(36)$ parameters. Therefore, because of these constraints, $V$ must have $72(36)-36(15)=36(21)$ parameters, consistent with the

number of $\frac{n(n-1)}{2}$ angles and $\frac{n(n+1)}{2}$ phases of a $n \times n$ unitary matrix. Since $\mathcal{M}^{\text {diag }}$ has $6(0)$ parameters, the r.h.s. of (37) has $36(21)$ (from $V)+6(0)=42(21)$, in consistency with the counting of parameters of the matrix $\mathcal{M}$ (137).

The unitarity of the matrix $V$ (39) implies [9, 10]

$$
\begin{aligned}
& K K^{\dagger}+R R^{\dagger}=1 \\
& S S^{\dagger}+T T^{\dagger}=1 \\
& K S^{\dagger}+R T^{\dagger}=0 \\
& K^{\dagger} K+S^{\dagger} S=1 \\
& R^{\dagger} R+T^{\dagger} T=1 \\
& K^{\dagger} R+S^{\dagger} T=0
\end{aligned}
$$

Let us do the exercise of counting again the number of parameters of the matrices $(K, R, S, T)$. If each of them were general complex, we would have for each 18(9) parameters, that gives for $(K, R, S, T)$ a total of $72(36)$ parameters. Relations (138) and (139) are hermitian, giving each 9(3) constraints, while (140) is general complex, giving 18(9) constraints. In total, we have again $9(3)+9(3)+18(9)=$ $36(15)$ constraints, and therefore, the set $(K, R, S, T)$ has $72(36)-36(15)=36(21)$ 
independent parameters, in agreement with the counting of independent parameters of the unitary matrix $V$.

On the other hand, the diagonalization of (137) reads

$$
\begin{aligned}
& K m_{L}^{\text {diag }} K^{t}+R M_{R}^{\text {diag }} R^{t}=m_{L} \\
& S m_{L}^{\text {diag }} S^{t}+T M_{R}^{\text {diag }} T^{t}=M_{R} \\
& K m_{L}^{\text {diag }} S^{t}+R M_{R}^{\text {diag }} T^{t}=m_{D}
\end{aligned}
$$

Verifying again the counting of parameters, we have for the r.h.s. of (144,146), $12(6)+12(6)+18(9)$ parameters from respectively $m_{L}, M_{R}$ and $m_{D}$. This gives a total of 42(21) independent parameters for the r.h.s., that is equal to the number of parameters of the 1.h.s., $36(21)+3(0)+3(0)$ from, respectively $(K, R, S, T), m_{L}^{\text {diag }}$ and $M_{R}^{\text {diag }}$.

\section{Acknowledgements}

We are grateful to Dr. V. Tello for reminding us the Keung-Senjanović effect 23] and for pointing out a theoretical study about the interesting possibility of measuring the neutrino Dirac mass matrix within the Left-Right model [47]. We are also indebted to Dr. P. Bhupal Dev for calling our attention to a recent updated formulation of flavor effects in leptogenesis [38].

\section{References}

[1] D. Falcone, Phys. Rev. D 68, 033002 (2003).

[2] E. Akhmedov, M. Frigerio and A. Smirnov, JHEP 0309, 021 (2003).

[3] E.K. Akhmedov and M. Frigerio, Phys. Rev. Lett. 96, 061802 (2006).

[4] F. Buccella, D. Falcone and L. Oliver, Phys. Rev. D 83, 093013 (2011).

[5] F. Buccella, D. Falcone, C.S. Fong, E. Nardi and G. Ricciardi, Phys. Rev. D 86, $035012(2012)$. 
[6] H. Georgi, in Particles and Fields, ed. C. Carlson (AIP, New York, 1975); H. Fritzsch and P. Minkowski, Ann. Phys. 93, 193 (1975).

[7] D. Falcone, Int. J. Mod. Phys. 17, 3981 (2002).

[8] J. Schechter and J. Valle, Phys. Rev. D 25, 774 (1982).

[9] J. Hashida, T. Morozumi and A. Purwanto, Prog. Theor. Phys. 103, 379 (2000, Erratum-ibid. 103, 865 (2000).

[10] G. Branco, T. Morozumi, B. Nobre and M. Rebelo, Nucl. Phys. B 617, 475 (2001).

[11] G. Branco, R. Gonzalez Felipe, F. Joaquim, M. Rebelo, Nucl. Phys. B 640, 202 (2002).

[12] G. Branco, R. Gonzalez Felipe and F. Joaquim, Rev. Mod. Phys. 84, 515 (2012).

[13] R. N. Mohapatra and J. C. Pati, Phys. Rev. D 11, 566 (1975).

[14] R. N. Mohapatra and J. C. Pati, Phys. Rev. D 11, 2558 (1975).

[15] P. Langacker and S. Sankar, Phys. Rev. D 40, 1569 (1989).

[16] P. Langacker, S. Sankar and K. Schilcher, Phys. Rev. D 38, 2841 (1988).

[17] J. C. Pati and A. Salam, Phys. Rev. D 10, 275 (1974) [Erratum-ibid. D 11, $703(1975)$.

[18] A. Broncano, M.B. Gavela and E. Jenkins, Phys. Lett. B 552, 177 (2003), Erratum, ibid. B 636, 330 (2006).

[19] K. Babu, B. Dutta and R. Mohapatra, Phys. Rev. D 67, 076006 (2003), and references therein.

[20] J.C. Helo et al., Phys. Rev. D 88, 011901 (2013).

[21] G. Aad (ATLAS Collaboration), JHEP 1110, 107 (2011).

[22] CMS Collaboration, CMS PAS EXO-12-017 (2012). 
[23] W.-Y. Keung and G. Senjanović, Phys. Rev. Lett. 50, 1427 (1983).

[24] N.G. Deshpande, J.F. Gunion, B. Kayser and F. Olness, Phys. Rev. D 44, 837 (1991).

[25] S. Wolfram, The Mathematica Book, 4th ed. (Wolfram Media/Cambridge University Press, 1999).

[26] D. Borah and M. Das, Phys. Rev. D 90, 015006 (2014).

[27] G. Branco, R. Gonzalez Felipe, F. Joaquim, I. Masina, M. Rebelo and C. Savoy, Phys. Rev. D 67, 073025 (2003).

[28] D. Falcone, Mod. Phys. Lett. A 26, 1375 (2011).

[29] J. Casas and A. Ibarra, Nucl. Phys. B 618, 171 (2001).

[30] A. Strumia, Lectures given at Les Houches Summer School on Theoretical Physics (August 2005) : Particle Physics Beyond the Standard Model, hep-ph/0608347.

[31] M. Fukugita and T. Yanagida, Phys. Lett. B 174, 45 (1986).

[32] For a review on baryogenesis via leptogenesis, see S. Davidson, E. Nardi and Y. Nir, Phys. Rept. 466, 105 (2008).

[33] A. Sakharov, JETP Lett. 91B, 24 (1967).

[34] R. Barbieri, P. Creminelli, A. Strumia and N. Tetradis, Nucl. Phys. B 575, 61 (2000).

[35] A. Abada, S. Davidson, F.X. Josse-Michaux, M. Losada and A. Riotto, JCAP 0604, 004 (2006).

[36] E. Nardi, Y. Nir, E. Roulet and J. Racker, JHEP 0601, 164 (2006).

[37] A. Abada, S. Davidson, A. Ibarra, F.X. Josse-Michaux, M. Losada and A. Riotto, JHEP 0609, 010 (2006).

[38] P. Bhupal Dev, P. Millington, A. Pilaftsis and D. Teresi, Nucl. Phys. B 886, 569 (2014). 
[39] L. Covi, E. Roulet and F. Vissani, Phys. Lett. B 384, 169 (1996).

[40] K. Babu, A. Bachri and H. Aissaoui, Nucl. Phys. B 738, 76 (2006).

[41] T. Fukuyama and N. Okada, Mod. Phys. Lett. A 17, 1725 (2002).

[42] M. Machacek, Nucl. Phys. B 159, 37 (1979).

[43] M.B. Gavela, A. Le Yaouanc, L. Oliver, O. Pène and J.-C. Raynal, Phys. Rev. D 23, 1580 (1981).

[44] L. Di Luzio, Aspects of symmetry breaking in Grand Unified Theories, arXiv:1110.3210, Chapter 1.

[45] H. Georgi and C. Jarlskog, Phys. Lett. B 86 (1979) 297.

[46] J.A. Harvey, P. Ramond and D.B. Reiss, Phys. Lett. B 92, 309 (1980).

[47] M. Nemevsek, G. Senjanović and V. Tello, Phys. Rev. Lett. 110, 151802 (2013). 